\title{
BASES FOR PSEUDOVARIETIES CLOSED UNDER BIDETERMINISTIC PRODUCT
}

\author{
ALFREDO COSTA AND ANA ESCADA
}

\begin{abstract}
We show that if $\mathrm{V}$ is a semigroup pseudovariety containing the finite semilattices and contained in DS, then it has a basis of pseudoidentities between finite products of regular pseudowords if, and only if, the corresponding variety of languages is closed under bideterministic product. The key to this equivalence is a weak generalization of the existence and uniqueness of J-reduced factorizations. This equational approach is used to address the locality of some pseudovarieties. In particular, it is shown that $\mathrm{DH} \cap \mathrm{ECom}$ is local, for any group pseudovariety $\mathrm{H}$.
\end{abstract}

\section{INTRODUCTION}

Reiterman's theorem [33] affirms that the pseudovarieties of semigroups are precisely the classes of finite semigroups defined by a basis of pseudoidentities between pseudowords. In this paper we refine this by showing that the basis may be chosen to consist solely of pseudoidentities between finite products of regular pseudowords, whenever $\mathrm{V}$ is a pseudovariety in the interval [SI, DS] that is closed under bideterministic product; motivated by this result, we call a finite product of regular pseudowords a multiregular pseudoword. Conversely, we give a proof that every pseudovariety of semigroups that has a basis of pseudoidentities between multiregular pseudowords is closed under bideterministic product; one may say that this converse is already hidden in the paper [31, where pseudovarieties closed under bideterministic product were first introduced, but note that neither in 31] nor in the sequels [15, 22, 16, 17] the profinite approach is explicitly present. In view of these results, one may argue that closure under bideterministic product is a relatively mild condition to impose upon a pseudovariety. Another reason for the interest in the bideterministic closure is that it is a natural companion of the closure under left deterministic product (which algebraically translates to the equality between $\mathrm{V}$ and the Mal'cev product

2010 Mathematics Subject Classification. 20M07, 20M05, 20M35, $18 \mathrm{~B} 40$.

Key words and phrases. Profinite semigroups; monoids; pseudovarieties; bideterministic product; basis of pseudoidentities; local pseudovarieties.

This work was partially supported by the Centre for Mathematics of the University of Coimbra - UID/MAT/00324/2019, funded by the Portuguese Government through FCT/MEC and co-funded by the European Regional Development Fund through the Partnership Agreement PT2020. 
$\mathrm{K}(\mathrm{m} \mathrm{V}$ ), the closure under right deterministic product (that translates to $\mathrm{V}=\mathrm{D}(\mathrm{m} \mathrm{V}$ ), and of the closure under unambiguous product (translated to $\mathrm{V}=\mathcal{L} \mathrm{I}(\mathrm{m} \mathrm{D})$.

Theorem 7.2, our main result, is the key to our refinement of Reiterman's theorem and other results. It is a sort of weak generalization of the theorem on the uniqueness of J-reduced factorizations [4], recalled as Theorem6.4. It is also a theorem in the spirit of the solutions of the "pseudoword problem" (of knowing when a pseudoidentity is satisfied by a pseudovariety) obtained in 37] for pseudovarieties closed under left, right or unambiguous product.

Some inspiration was taken from the fact, shown in [7], that the free profinite semigroups over $\mathrm{V}$ are equidivisible when $\mathrm{V}$ is closed under unambiguous product. Here, we show that a weak form of equidivisibility still stands when we only know that $\mathrm{V}$ is closed under bideterministic product (Theorem 4.9). This is crucial for the proof of our main result. This form of weak divisibility is based on the notion of good factorization, which defines the Pin-Thérien expansion, first introduced in [31.

The property of a pseudovariety of semigroups being local is relevant but often difficult to prove. In [19] it is shown that if $\mathrm{V}$ is a local monoidal pseudovariety of semigroups containing SI, then $\mathrm{K} m \mathrm{~V}$ and $\mathrm{D}(\mathrm{m} \mathrm{V}$ are also local monoidal pseudovarieties of semigroups. Consider now the operator $\mathrm{V} \mapsto \overline{\mathrm{V}}$ that associates to each pseudovariety of semigroups $\mathrm{V}$ the least pseudovariety of semigroups $\bar{V}$ containing $V$ that is closed under bideterministic product. The methods used in [19] do not carry on to this operator (see the discussion in Section 10). But, restricting our attention to the class RS of finite semigroups whose set of regular elements is a subsemigroup, then, with our key result (Theorem [7.2) we do prove that if $\mathrm{V}$ is a local monoidal pseudovariety of semigroups contained in the interval [SI, DS $\cap \mathrm{RS}$ ], then $\bar{V}$ is also a local monoidal pseudovariety of semigroups. This implies, for example, that $\mathrm{DH} \cap \mathrm{ECom}$ is local, for every pseudovariety $\mathrm{H}$ of groups (this family of peudovarieties has received some attention [11, 10, 13]).

The paper is organized as follows. After the introduction and a section of preliminaries, we recall in Section 3 the Pin-Thérien expansion of a monoid, also giving its semigroup counterpart. The latter is because we want to work with semigroup pseudovarieties that are non-monoidal, such as those of the form $\mathrm{V} * \mathrm{D}$, seen in Section 10. Sections 4 to 7 constitute the paper's core, where several aspects of the notion of good factorization of a pseudoword are explored, culminating in the main results. Finally, Sections 8 to 10 are motivated by the investigation on the locality of pseudovarieties.

\section{Preliminaries}

For more details on (profinite) semigroups the reader is referred to the introductory text [3] and the books [1, 34]. The definitions and results related with monoids are similar. 
For a semigroup $S$, the monoid $S^{I}=S \uplus\{1\}$ is obtained from $S$ by adjoining to $S$ a neutral element 1 not in $S$. Every semigroup homomorphism $\varphi: S \rightarrow T$ admits an extension to a monoid homomorphism $\varphi^{I}: S^{I} \rightarrow T^{I}$ such that $\varphi^{I}(1)=1$. The object $S^{I}$ may be different from the frequently used $S^{1}$ : the latter equals $S$ if $S$ is a monoid, and is $S^{I}$ if $S$ is not a monoid.

We use the standard notations for Green's equivalence relations $\mathcal{R}, \mathcal{L}$ and $\mathcal{J}$ and its associated quasi-orders $\leq_{\mathcal{R}}, \leq_{\mathcal{L}}$ and $\leq_{\mathcal{J}}$ on a semigroup $S$ : for $s, t \in S, s \leq_{\mathcal{R}} t$ if $s \in t S^{I}, s \leq_{\mathcal{L}} t$ if $s \in S^{I} t$, an $s \leq_{\mathcal{J}} t$ if $s \in S^{I} t S^{I}$, and for $\mathcal{K} \in\{\mathcal{R}, \mathcal{L}, \mathcal{J}\}$, we have $s \mathcal{K} t$ when $s \leq_{\mathcal{K}} t$ and $t \leq_{\mathcal{K}} s$. The elements $s \in S$ such that $s \in s S s$ are said to be regular. In general, the set of regular elements of a semigroup is not a subsemigroup. If $S$ is a compact semigroup (i.e., a semigroup endowed with a compact topology for which the semigroup operation is continuous), then, for each $\mathcal{K} \in\{\mathcal{R}, \mathcal{L}, \mathcal{J}\}$, a $\mathcal{K}$-class $K$ of $S$ contains a regular element if and only if all its elements are regular, in which case we say that $K$ is regular. Moreover, one also views $S^{I}$ as a compact semigroup by adding $I$ has an isolated point.

2.1. Pseudovarieties. A pseudovariety of semigroups is a class of finite semigroups closed under taking subsemigroups, homomorphic images and finitary direct products. The pseudovariety of all finite semigroups is denoted by S. We list some other pseudovarieties which have a role in this paper: SI, the pseudovariety of all finite semillatices; J, the pseudovariety of all finite semigroups whose regular $\mathcal{J}$-classes are trivial; $\mathrm{D}$, the pseudovariety of all finite semigroups whose idempotents are right zeros; and $\mathrm{K}$, the dual of $\mathrm{D}$. For any pseudovariety $\mathrm{V}$, one denotes by $\mathcal{L} \mathrm{V}$ the pseudovariety of all finite semigroups $S$ such that $e S e \in \mathrm{V}$, for all idempotents $e \in S$, and DV denotes the pseudovariety of all finite semigroups $S$ whose regular $\mathcal{J}$-classes are semigroups that belong to $\mathrm{V}$. As we have mentioned in the introduction, the pseudovariety DS will play a special role in this paper: quite frequently, in the study of pseudovarieties, one has to consider the cases $\mathrm{V} \subseteq \mathrm{DS}$ and $\mathrm{V} \nsubseteq \mathrm{DS}$ separately.

Let $\mathrm{W}$ and $\mathrm{V}$ be pseudovarieties of semigroups. The Mal'cev product $\mathrm{W} m \mathrm{~V}$ is the pseudovariety of semigroups generated by finite semigroups $S$ for which there is a semigroup homomorphism $\varphi: S \rightarrow T$, for some $T \in \mathrm{V}$, such that $\varphi^{-1}(e) \in \mathrm{W}$, for each idempotent $e \in T$. The semidirect product $\mathrm{V} * \mathrm{~W}$ is the pseudovariety generated by all semidirect products of the form $S * T$ with $S \in \mathrm{V}$ and $T \in \mathrm{W}$.

2.2. Free pro- $V$ semigroups. In what follows, finite semigroups are viewed as compact semigroups, endowed with the discrete topology. A compact semigroup $S$ is said to be A-generated if there is a map $\iota: A \rightarrow S$ such that $\iota(A)$ generates a dense subsemigroup of $S$. It is said to be residually in $\mathrm{V}$, where $\mathrm{V}$ is a pseudovariety, if for every two distinct elements $s_{1}$ and $s_{2}$ of $S$, there is some continuous homomorphism $\varphi: S \rightarrow T$ into a semigroup $T \in \mathrm{V}$ such that $\varphi\left(s_{1}\right) \neq \varphi\left(s_{2}\right)$. By a pro-V semigroup we mean a compact semigroup residually in $\mathrm{V}$. 
In this paper all alphabets are finite. For each alphabet $A$, there is a unique (up to isomorphism) $A$-generated free pro- $\mathrm{V}$ semigroup, denoted $\bar{\Omega}_{A} \mathrm{~V}$, endowed with a mapping $\iota \mathrm{V}: A \rightarrow \bar{\Omega}_{A} \mathrm{~V}$, which satisfies the following universal property: for any map $\varphi: A \rightarrow S$ into a pro-V semigroup $S$ there is a unique continuous homomorphism $\widehat{\varphi}^{\vee}: \bar{\Omega}_{A} \mathrm{~V} \rightarrow S$ such that $\hat{\varphi}^{\vee} \iota_{\mathrm{V}}=\varphi$. The finiteness of $A$ guarantees that $\bar{\Omega}_{A} \vee$ is metrizable. The elements of $\bar{\Omega}_{A} \mathrm{~V}$ are called pseudowords (with respect to $\mathrm{V}$ ). In particular, the map $\iota \mathrm{V}: A \rightarrow \bar{\Omega}_{A} \mathrm{~V}$ induces a unique continuous homomorphism $p_{\mathrm{V}}: \bar{\Omega}_{A} \mathrm{~S} \rightarrow \bar{\Omega}_{A} \mathrm{~V}$, which is called the natural projection of $\bar{\Omega}_{A} \mathrm{~S}$ onto $\bar{\Omega}_{A} \mathrm{~V}$, and satisfies $p_{\mathrm{V}} \circ \iota_{\mathrm{S}}=\iota_{\mathrm{V}}$. We write $[u]_{\mathrm{V}}$ instead of $p_{\mathrm{V}}(u)$. In case $\mathrm{V}=\mathrm{S}$, we use the notation $\widehat{\varphi}$ for $\widehat{\varphi}^{\mathrm{S}}$, and we speak of profinite semigroups instead of pro-S semigroups.

Let $\Omega_{A} \mathrm{~V}$ be the subsemigroup of $\bar{\Omega}_{A} \mathrm{~V}$ generated by $\iota \mathrm{V}(A)$. If $\mathrm{V}$ is not the trivial pseudovariety, then $\iota_{\mathrm{V}}$ is injective, and so $A$ may actually be seen as a subset of $\bar{\Omega}_{A} \mathrm{~V}$. Moreover, if $\mathrm{V}$ contains the pseudovariety $\mathrm{N}$ of finite nilpotent semigroups, then $\Omega_{A} \mathrm{~V}$ is naturally isomorphic to the free semigroup $A^{+}$, endowed with the discrete topology, and the elements of $\Omega_{A} \mathrm{~V}$ are isolated in $\bar{\Omega}_{A} \mathrm{~V}$. From hereon, we identify $\Omega_{A} \mathrm{~V}$ with $A^{+}$when $\mathrm{V}$ contains $\mathrm{N}$.

Let $\mathrm{V}$ be a pseudovariety that contains $\mathrm{SI}$ and let $c: \bar{\Omega}_{A} \mathrm{~V} \rightarrow \bar{\Omega}_{A} \mathrm{SI}$ be the content mapping, the unique continuous homomorphism from $\bar{\Omega}_{A} \mathrm{~V}$ onto $\bar{\Omega}_{A} \mathrm{SI}$ such that $c \circ \iota_{\mathrm{V}}=\iota_{\mathrm{SI}}$. If $u$ is a word on $A$, then $c(u)$ is the set of letters occurring in $u$. In general, $c(u)$ is the content of $u$, for every $u \in \bar{\Omega}_{A} \mathrm{~V}$.

A pseudoidentity $(u=v)$ in variables of the alphabet $A$ is a formal equality between elements $u$ and $v$ of $\bar{\Omega}_{A}$ S. For a profinite semigroup $S$, we write $S \models u=v$ when $S$ satisfies the pseudoidentity $u=v$ (that is, for every map $\varphi: A \rightarrow S$, we have $\widehat{\varphi}(u)=\widehat{\varphi}(v))$, and write $\mathrm{V} \models u=v$ when all semigroups of $\mathrm{V}$ satisfy $u=v$. More generally, we write $\mathrm{V} \models \Sigma$ when $\Sigma$ is a set of pseudoidentities satisfied by all semigroups of $\mathrm{V}$. The class of finite semigroups satisfying all elements of $\Sigma$ is denoted $\llbracket \Sigma \rrbracket$. Reiterman's Theorem [33] states that the pseudovarieties of semigroups are precisely the classes of the form $\llbracket \Sigma \rrbracket$. As a relevant example, we have DS $=\llbracket\left((x y)^{\omega}(y x)^{\omega}(x y)^{\omega}\right)^{\omega}=(x y)^{\omega} \rrbracket$, where, if $s$ is an element of a profinite semigroup, $s^{\omega}$ is the idempotent $s^{\omega}=\lim s^{n !}$ (more generally, we use the notation $s^{\omega+k}=\lim s^{n !+k}$ ). One says that $\Sigma$ is a basis for $\mathrm{V}$ when $\mathrm{V}=\llbracket \Sigma \rrbracket$.

A language $L \subseteq A^{+}$is $\mathrm{V}$-recognizable if there is a homomorphism $\varphi$ from $A^{+}$into a semigroup $S$ of $\mathrm{V}$ such that $L=\varphi^{-1} \varphi L$. The following proposition establishes a link between $\mathrm{V}$-recognizable languages and the topology of $\bar{\Omega}_{A} \mathrm{~V}$, when $\mathrm{V}$ contains $\mathrm{N}$. The restriction $\mathrm{V} \supseteq \mathrm{N}$ may be dropped, but the statement becomes less direct, and it suffices for us that $\mathrm{V} \supseteq \mathrm{N}$.

Theorem 2.1 (cf. [1, Theorem 3.6.1]). Let $\mathrm{V}$ be a semigroup pseudovariety containing N. A language $L \subseteq A^{+}$is $\mathrm{V}$-recognizable if and only if its closure $\bar{L}$ in $\bar{\Omega}_{A} \vee$ is open.

Note that, in Theorem 2.1, one has $\bar{L} \cap A^{+}=L$, because the elements of $A^{+}$are isolated in $\bar{\Omega}_{A} \mathrm{~V}$. Hence, Theorem 2.1 states that the $\mathrm{V}$-recognizable 
languages of $A^{+}$are precisely the traces in $A^{+}$of the clopen subsets of $\bar{\Omega}_{A} \mathrm{~V}$. We shall use Theorem 2.1 abundantly, without reference.

Theorem 2.1 is relevant in the framework of Eilenberg's Theorem 21 on the correspondence between a semigroup pseudovariety $\mathrm{V}$ and the variety $\mathcal{V}$ of +-languages that are $\mathrm{V}$-recognizable (recall that a +-language is a subset of a free semigroup, while a $*$-language is a subset of a free monoid). For the sake of conciseness, we write $L \in \mathcal{V}$ whenever $L$ is a $\mathrm{V}$-recognizable language of $A^{+}$, instead of the more precise $L \in A^{+} \mathcal{V}$.

We shall frequently switch from the viewpoint of +-languages and semigroup pseudovarieties to that of $*$-languages and monoid pseudovarieties.

2.3. Marked products. Our departing point is the following definition, whose first three items are nowadays classical [28].

Definition 2.2. Let $L$ and $K$ be languages of $A^{*}$, and let $a \in A$. The language $L a K$, viewed as product of the languages $L,\{a\}$ and $K$ - usually referred to as a marked product of $L$ and $K$ - is said to be:

(1) an unambiguous product when every element $u$ of $L a K$ has a unique factorization $u=u_{1} a u_{2}$ such that $u_{1} \in L$ and $u_{2} \in K$;

(2) a left deterministic product when every word of LaK has a unique prefix in $L a$;

(3) a right deterministic product when every word of LaK has a unique suffix in $a K$;

(4) a bideterministic product if the marked product LaK is simultaneously right and left deterministic.

We say that a language $L$ of $A^{*}$ is a prefix code (resp. suffix code) if $\forall u \in L, u A^{+} \cap L=\emptyset$ (resp. $\forall u \in L, A^{+} u \cap L=\emptyset$ ).

Note that $L a K$ is left deterministic if and only if $L a$ is a prefix code. Dually, it is right deterministic if and only if $a K$ is a suffix code.

Next, we introduce a varietal companion of Definition 2.2 .

Definition 2.3. Let $V$ be a pseudovariety of monoids, and let $\mathcal{V}$ be the correspondent variety of $\mathrm{V}$-recognizable $*$-languages. Then $\mathrm{V}$ is said to be:

(1) closed under unanbiguous product if $L a K \in \mathcal{V}$ whenever $L, K \in \mathcal{V}$, $a$ is a letter and $L a K$ is an unambiguous product;

(2) closed under left deterministic product if $L a K \in \mathcal{V}$ whenever $L, K \in \mathcal{V}$, $a$ is a letter and $L a$ is a prefix code;

(3) closed under right deterministic product if $L a K \in \mathcal{V}$ whenever $L, K \in \mathcal{V}$, $a$ is a letter and $a K$ is a suffix code;

(4) closed under bideterministic product if $L a K \in \mathcal{V}$ whenever $L, K \in \mathcal{V}$, $a$ is a letter, $L a$ is a prefix code and $a K$ is a suffix code.

It is well known that we have the following characterization of the first three types of pseudovarieties mentioned in Definition 2.3.

Theorem 2.4 ([27, 28, 30, 35, see also survey [29]). Let $\mathrm{V}$ be a pseudovariety of monoids. Then: 
(1) $\mathrm{V}$ is closed under unambiguous product if and only if $\mathrm{V}=\mathcal{L} \mathrm{I}(\mathrm{m} \mathrm{V}$;

(2) $\mathrm{V}$ is closed under left deterministic product if and only if $\mathrm{V}=\mathrm{K}(\mathrm{V} \mathrm{V}$;

(3) $\mathrm{V}$ is closed under right deterministic product if and only if $\mathrm{V}=\mathrm{D} / \mathrm{V}$.

On the other hand, pseudovarieties closed under bidetermistic product have no characterization that, like in Theorem 2.4, uses a Mal'cev product.

In this section, we have so far stayed in the realm of $*$-languages, and in the corresponding one of monoid pseudovarieties. But the definitions and results we reviewed have natural companions in the realms of +-varieties and semigroup pseudovarieties. A way to define these counterparts is by restricting each language $L$ and $K$ to be either a +-language or the language $\{1\}$. We next see a concrete manifestation of this in the case of the bidetermistic product, the subject of attention in this paper.

Definition 2.5. Let $\mathrm{V}$ be a semigroup pseudovariety, and $\mathcal{V}$ be the variety of $\mathrm{V}$-recognizable +-languages. Then $\mathrm{V}$ is closed under bideterministic product if $L a K \in \mathcal{V}$ when $a$ is a letter and the next conditions are satisfied: $L \in \mathcal{V}$ or $L=\{1\} ; K \in \mathcal{V}$ or $K=\{1\} ; L a$ is a prefix code; $a K$ is a suffix code.

For $u \in A^{*}$, let $\mathrm{i}_{1}(u)$ be the first letter of $u$ if $u \neq 1$, and $\mathrm{i}_{1}(1)=1$. Dually, $\mathrm{t}_{1}(u)$ is the last letter of $u$ if $u \neq 1$, and $\mathrm{t}_{1}(1)=1$. The maps $\mathrm{i}_{1}$ and $\mathrm{t}_{1}$ extend uniquely to continuous maps from $\left(\bar{\Omega}_{A} \mathrm{~S}\right)^{1}$ into $A \cup\{1\}$.

Remark 2.6. The product of prefix codes is a prefix code. In particular, $L u$ is a prefix code when $L$ is a prefix code, whenever $u \in A^{*}$. Dual remarks hold for suffix codes. This implies that, still assuming that $V$ is closed under bideterministic product and that $\mathcal{V}$ is its corresponding variety of +languages, if $L_{1} L_{2}, \ldots, L_{n} \in \mathcal{V}$, and $u_{0}, u_{1}, \ldots, u_{n} \in A^{*}$, with $u_{i} \neq 1$ when $i \neq 0$ and $i \neq n$, are such that $L_{i} \cdot \mathrm{i}_{1}\left(u_{i}\right)$ is a prefix code and $\mathrm{t}_{1}\left(u_{i-1}\right) \cdot L_{i}$ is a suffix code for every $i \in\{1, \ldots, n\}$, then $u_{0} L_{1} u_{1} L_{2} u_{2} \cdots u_{n-1} L_{n} u_{n} \in \mathcal{V}$.

\section{The Pin-ThÉRIEn EXPAnsion}

3.1. The Pin-Thérien expansion of a finite monoid. In what follows, we consider an onto homomorphism of monoids $\varphi: A^{*} \rightarrow M$ such that $M$ is finite. The finiteness of $M$ is frequently not important (sometimes it is, like in Theorem 5.4), but that is the framework in which we are interested, and it is a general assumption also made in [31.

Definition 3.1. A good factorization (with respect to $\varphi$ ) is a triple $\left(x_{0}, a, x_{1}\right)$ of $A^{*} \times A \times A^{*}$ such that $\varphi\left(x_{0} a\right)<_{\mathcal{R}} \varphi\left(x_{0}\right)$ and $\varphi\left(a x_{1}\right)<_{\mathcal{L}} \varphi\left(x_{1}\right)$. Two good factorizations $\left(x_{0}, a, x_{1}\right)$ and $\left(y_{0}, b, y_{1}\right)$ are said to be equivalent when $\varphi\left(x_{0}\right)=\varphi\left(y_{0}\right), a=b$ and $\varphi\left(x_{1}\right)=\varphi\left(y_{1}\right)$. A good factorization of $x \in A^{*}$ is a good factorization $\left(x_{0}, a, x_{1}\right)$ such that $x=x_{0} \cdot a \cdot x_{1}$.

It is shown in [16] that, for all $x, y \in A^{*}$, every good factorization of $x$ is equivalent to at most one good factorization of $y$.

Definition 3.2. Let $\sim_{\varphi}$ be the relation on $A^{*}$ defined by $x \sim_{\varphi} y$ if and only if the following conditions are satisfied: 
(1) $\varphi(x)=\varphi(y)$;

(2) each good factorization of $x$ is equivalent to a good factorization of $y$;

(3) each good factorization of $y$ is equivalent to a good factorization of $x$.

The relation $\sim_{\varphi}$ is a congruence [31].

Definition 3.3. Denote $A^{*} / \sim_{\varphi}$ by $M_{\varphi}$, and by $\varphi_{\mathrm{bd}}$ the corresponding quotient morphism from $A^{*}$ onto $A^{*} / \sim_{\varphi}$. We say that $M_{\varphi}$ is the Pin-Thérien expansion of $M$ with respect to $\varphi$.

Note that there is a unique onto monoid homomorphism $p_{\varphi}: M_{\varphi} \rightarrow M$ such that $\varphi=p_{\varphi} \circ \varphi_{\mathrm{bd}}$. Note also that the finiteness of $M$ guarantees the finiteness of $M_{\varphi}$ [31]. The correspondence $\varphi \mapsto \varphi_{\mathrm{bd}}$ is indeed an expansion cut to generators in the sense of Birget and Rhodes, as shown in [31. In fact, it is proved in [16] that it is an expansion in a broader sense.

Definition 3.4. For a monoid pseudovariety $\mathrm{V}$, denote by $\mathrm{V}_{\mathrm{bd}}$ the monoid pseudovariety generated by Pin-Thérien expansions of monoids in $\mathrm{V}$. We say that $\mathrm{V}$ is closed under Pin-Thérien expansion when $\mathrm{V}=\mathrm{V}_{\mathrm{bd}}$.

Theorem 3.5 ([31, Corollary 4.5]). Let $\mathrm{V}$ be a monoid pseudovariety. Then $\mathrm{V}=\mathrm{V}_{\mathrm{bd}}$ if and only if $\mathrm{V}$ is closed under bideterministic product.

The intersection of a family of monoid pseudovarieties closed under bideterministic product is a pseudovariety closed under bideterministic product. Hence, for each monoid pseudovariety $\mathrm{V}$ we may consider the least monoid pseudovariety $\overline{\mathrm{V}}$ closed under bideterministic product and containing $\mathrm{V}$.

Remark 3.6. Consider the chain of pseudovarieties $\left(\mathrm{V}_{n}\right)_{n \geq 0}$ recursively defined by $\mathrm{V}_{0}=\mathrm{V}$ and $\mathrm{V}_{n}=\left(\mathrm{V}_{n-1}\right)_{\text {bd }}$ for each $n \geq 1$. Then $\bigcup_{n \geq 0} \mathrm{~V}_{n}$ is closed for the bideterministic product (cf. Theorem 3.5) and $\bar{V}=\bigcup_{n \geq 0} \vee_{n}$.

Example 3.7. It is easy to see that the Pin-Thérien expansion of a finite group $G$, viewed as a monoid, is $G$ itself. Hence, viewing a pseudovariety of groups $\mathrm{H}$ as a monoid pseudovariety, one has $\mathrm{H}=\mathrm{H}_{\mathrm{bd}}=\overline{\mathrm{H}}$.

Example 3.8. Let Ecom be the pseudovariety of monoids whose idempotents commute. In [31] it is shown that $\overline{\mathrm{SI} \vee \mathrm{H}}=\mathrm{DH} \cap \mathrm{ECom}$, whenever $\mathrm{H}$ is a pseudovariety of groups. In particular, the equality $\overline{\mathrm{SI}}=\mathrm{J} \cap \mathrm{ECom}$ holds.

For a pseudovariety $\mathrm{V}$ of monoids, we denote by $\mathrm{RV}$ the class of finite monoids whose set of regular elements is a submonoid in $\mathrm{V}$. When $\mathrm{V} \subseteq \mathrm{CR}$, $\mathrm{RV}$ is a pseudovariety, where $\mathrm{CR}$ is the pseudovariety of all finite monoids which are completely regular.

Example 3.9. If $\mathrm{V} \subseteq \mathrm{CR}$, then $\overline{\mathrm{V}}=\mathrm{R} \mathrm{V} \cap \llbracket x^{\omega}(x y)^{\omega}=(x y)^{\omega}=(x y)^{\omega} y^{\omega} \rrbracket$. This formula was deduced in [22] from its special case, proved in [15], in which $\mathrm{V} \subseteq \mathrm{B}$. 
3.2. The Pin-Thérien expansion of a finite semigroup. In the following lines, we define a semigroup version of the Pin-Thérien expansion. Let $\varphi: A^{+} \rightarrow S$ be an onto homomorphism of semigroups, with $S$ finite. Consider the monoid homomorphism $\varphi^{I}: A^{*} \rightarrow S^{I}$ such that $\varphi^{I}(u)=\varphi(u)$, when $u \neq 1$. Then the empty word 1 of $A^{*}$ is the unique element of the $\sim_{\varphi^{I}}$-class of 1 . Therefore, if $I^{\prime}=\left(\varphi^{I}\right)_{\mathrm{bd}}(1)$ is the identity of $\left(S^{I}\right)_{\varphi^{I}}$, then the semigroup $S_{\varphi}=\left(\varphi^{I}\right)_{\mathrm{bd}}\left(A^{+}\right)$satisfies $S_{\varphi}=\left(S^{I}\right)_{\varphi^{I}} \backslash\left\{I^{\prime}\right\}$, and so

$$
\left(S_{\varphi}\right)^{I}=\left(S^{I}\right)_{\varphi^{I}}
$$

where we are making the identification $I=I^{\prime}$. We may then consider the semigroup homomorphism $\varphi_{\mathrm{bd}}: A^{+} \rightarrow S_{\varphi}$ obtained by the restriction of $\left(\varphi^{I}\right)_{\text {bd }}$ to $A^{+}$. Note that

$$
\left(\varphi_{\mathrm{bd}}\right)^{I}=\left(\varphi^{I}\right)_{\mathrm{bd}}
$$

The semigroup $S_{\varphi}$ is the (semigroup) Pin-Thérien expansion of $S$ with respect to $\varphi$. We then let $\sim_{\varphi}$ be the kernel of $\varphi_{\mathrm{bd}}$. We also denote by $p_{\varphi}$ the unique onto semigroup homomorphism $p_{\varphi}: S_{\varphi} \rightarrow S$ such that $\varphi=p_{\varphi} \circ \varphi_{\text {bd }}$. Note also that, for such a $\varphi$, we have $\left(p_{\varphi}\right)^{I}=p_{\varphi^{I}}$.

Remark 3.10. The expansion $\varphi_{\mathrm{bd}}: A^{+} \rightarrow S_{\mathrm{bd}}$ may equivalently be defined by adapting the definitions given in Subsection 3.1, by letting $\left(x_{0}, a, x_{1}\right)$ be a good factorization of $x_{0} a x_{1} \in A^{+}$whenever $\varphi\left(x_{0} a\right)<_{\mathcal{R}} \varphi^{I}\left(x_{0}\right)$ and $\varphi\left(a x_{1}\right)<_{\mathcal{L}} \varphi^{I}\left(x_{1}\right)$.

For a pseudovariety of monoids $\mathrm{V}$, one denotes by $\mathrm{V}_{\mathrm{S}}$ the least pseudovariety of semigroups containing $\mathrm{V}$. It is well known that $S \in \mathrm{V}_{\mathrm{S}}$ if and only if $S^{1} \in \mathrm{V}$. Moreover, if $\mathrm{V}$ contains $\mathrm{SI}$, then $S \in \mathrm{V}_{\mathrm{S}}$ if and only if $S^{I} \in \mathrm{V}$, a fact that we shall use in the proof of the next proposition.

Proposition 3.11. If $\mathrm{V}$ is a pseudovariety of monoids containing $\mathrm{SI}$, then the equality $\left(\mathrm{V}_{\mathrm{S}}\right)_{\mathrm{bd}}=\left(\mathrm{V}_{\mathrm{bd}}\right)_{\mathrm{S}}$ holds.

Proof. Let $\psi: A^{*} \rightarrow M$ be a surjective homomorphism onto a monoid $M$ of $\mathrm{V}$. The proof of $\left(\mathrm{V}_{\mathrm{S}}\right)_{\mathrm{bd}} \supseteq \mathrm{V}_{\mathrm{bd}}$, equivalently of $\left(\mathrm{V}_{\mathrm{S}}\right)_{\mathrm{bd}} \supseteq\left(\mathrm{V}_{\mathrm{bd}}\right)_{\mathrm{S}}$, is concluded once we show that $M_{\psi}$, viewed as a semigroup, belongs to $\left(\mathrm{V}_{\mathrm{S}}\right)_{\mathrm{bd}}$.

Denote by $1_{M}$ the identity of $M$. We consider two cases.

Suppose first that $\psi^{-1}\left(1_{M}\right)=\{1\}$. Let $B=A \uplus\{b\}$, where $b$ is a new letter not in $A$. Consider the homomorphism $\lambda_{b}: B^{*} \rightarrow A^{*}$ such that $\lambda_{b}(b)=1$ and $\lambda_{b}(a)=a$ for every $a \in A$. Denote $\lambda_{b}(u)$ by $\bar{u}$. Consider also the semigroup homomorphism $\psi_{b}: B^{+} \rightarrow M$ such that $\psi_{b}(b)=1_{M}$ and $\psi_{b}(a)=\psi(a)$. We claim that, in the category of semigroups, $M_{\psi}$ is a homomorphic image of $M_{\psi_{b}}$. For that purpose, we collect the following series of facts:

(1) We have $b^{+}=\psi_{b}^{-1}\left(1_{M}\right)$ and $\{1\}=\psi^{-1}\left(1_{M}\right)$, thus $b^{+} \backslash\{b\}$ and $\{b\}$ are $\sim_{\psi_{b}}$-classes, and $\{1\}$ is a $\sim_{\psi^{-c l a s s}}$.

(2) If $\left(x_{0}, a, x_{1}\right) \in B^{*} \times B \times B^{*}$ is a good factorization with respect to $\psi_{b}$ of an element of $B^{+} \backslash b$, then $a \neq b$. 
(3) Let $\left(x_{0}, a, x_{1}\right) \in B^{*} \times A \times B^{*}$. Then $\left(x_{0}, a, x_{1}\right)$ is a good factorization with respect to $\left(\psi_{b}\right)^{I}$ if and only if $\left(\overline{x_{0}}, a, \overline{x_{1}}\right)$ is a good factorization with respect to $\psi$. This is trus because $\left(\psi_{b}\right)^{I}\left(x_{0}\right)=\psi\left(\overline{x_{0}}\right)$ unless $x_{0}=1$, in which case $\left(\psi_{b}\right)^{I}(1 \cdot a)<_{\mathcal{R}}\left(\psi_{b}\right)^{I}(1)=I$ and $\psi(1 \cdot a)<_{\mathcal{R}}$ $\psi(1)=1_{M}$ hold, and because of the dual phenomena concerning the third component of the factorizations.

Taking into account the partition $B^{+}=\{b\} \uplus\left(b^{+} \backslash\{b\}\right) \uplus B^{*} A B^{*}$, it follows that the map from $M_{\psi_{b}}=B^{+} / \sim_{\psi_{b}}$ to $M_{\psi}=A^{*} / \sim_{\psi}$, sending $u / \sim_{\psi_{b}}$ to $\bar{u} / \sim_{\psi}$, is a well defined onto homomorphism of semigroups, thus establishing the claim. As $M \in \mathrm{V}_{\mathrm{S}}$ and $M_{\psi_{b}} \in\left(\mathrm{V}_{\mathrm{S}}\right)_{\mathrm{bd}}$, it follows that $M_{\psi} \in\left(\mathrm{V}_{\mathrm{S}}\right)_{\mathrm{bd}}$.

Suppose now that $\psi^{-1}\left(1_{M}\right) \neq\{1\}$. Then $\psi\left(A^{+}\right)=M$. Let $\Psi$ be the monoid homomorphism from $A^{*}$ onto $M^{I}$ such that $\Psi(u)=\psi(u)$ for every $u \in A^{+}$. Note that $\Psi$ is onto and that $\Psi^{-1}(I)=\{1\}$. Moreover, since $\mathrm{SI} \subseteq \mathrm{V}$, the monoid $M^{I}$ belongs to $\mathrm{V}$. Hence, by the already proved case, we have $\left(M^{I}\right)_{\Psi} \in\left(\mathrm{V}_{\mathrm{S}}\right)_{\mathrm{bd}}$. Let $\pi$ be the onto monoid homomorphism from $M^{I}$ to $M$ whose restriction to $M$ is the identity. Then $\pi \circ \Psi=\psi$. Because we are dealing with an expansion cut to generators, this implies that $M_{\psi}$ is a homomorphic image of $\left(M^{I}\right)_{\Psi}$, whence $M_{\psi} \in\left(\mathrm{V}_{\mathrm{S}}\right)_{\mathrm{bd}}$.

Finally, we show that $\left(\mathrm{V}_{\mathrm{S}}\right)_{\text {bd }} \subseteq\left(\mathrm{V}_{\mathrm{bd}}\right)_{\mathrm{s}}$. Let $S \in \mathrm{V}_{\mathrm{S}}$, and let $\varphi: A^{+} \rightarrow S$ be an onto homomorphism. Since $\mathrm{V}$ contains $\mathrm{SI}$, we have $S^{I} \in \mathrm{V}$, whence $\left(S^{I}\right)_{\varphi^{I}} \in \mathrm{V}_{\mathrm{bd}}$. But $\left(S^{I}\right)_{\varphi^{I}}=\left(S_{\varphi}\right)^{I}$, whence $S_{\varphi} \in\left(\mathrm{V}_{\mathrm{bd}}\right)_{\mathrm{S}}$.

We omit the proof of the following theorem, since it can be made by just imitating the proof in [31] of its monoid analog (Theorem 3.5).

Theorem 3.12. A pseudovariety of semigroups $\mathrm{V}$ satisfies $\mathrm{V}=\mathrm{V}_{\mathrm{bd}}$ if and only if $\mathrm{V}$ is closed under bideterministic product.

As for monoids, let $\bar{V}$ be the least semigroup pseudovariety, containing the semigroup pseudovariety $\mathrm{V}$, which is closed under bideterministic product. Note that Remark 3.6 also holds for pseudovarieties of semigroups. This fact and Proposition 3.11 yeld the following corollary.

Corollary 3.13. If $\mathrm{V}$ is a monoid pseudovariety containing $\mathrm{SI}$, then the equality $\overline{\mathrm{V}}_{\mathrm{S}}=\overline{\mathrm{V}}_{\mathrm{S}}$ holds.

In Corollary 3.13, the hypothesis $\mathrm{V} \supseteq \mathrm{SI}$ is needed as seen below.

Example 3.14. Let I be the class of trivial semigroups. Viewing I as a pseudovariety of monoids, one has $\bar{I}=\mathrm{I}$ (Example 3.7), but if we view I as a pseudovariety of semigroups, then we get $\bar{I}=\mathrm{N}$. Indeed, $\bar{I} \subseteq \mathrm{N}$ because an N-recognizable language is either finite or co-finite, and since a co-finite language can neither be a prefix code nor a suffix code, one clearly has that $\mathrm{N}$ is closed under bideterministic product. On the other hand, $\mathrm{N} \subseteq \overline{\mathrm{I}}$ since every finite language is the finite union of bideterministic products of the form $\{1\} \cdot a_{1} \cdot\{1\} \cdot a_{2} \cdots a_{n-1} \cdot\{1\} \cdot a_{n}\{1\}$, with $a_{1}, \ldots, a_{n}$ letters. 


\section{Good FACTORIZATIONS OF PSEUdOWORdS}

Inspired by the concept of good factorization of a word, we define an analog for pseudowords.

Definition 4.1. Let $\mathrm{V}$ be a pseudovariety of semigroups. A good factorization of an element $x$ of $\bar{\Omega}_{A} \mathrm{~V}$ is a triple $(\pi, a, \rho)$ in $\left(\bar{\Omega}_{A} \mathrm{~V}\right)^{1} \times A \times\left(\bar{\Omega}_{A} \mathrm{~V}\right)^{1}$, with $x=\pi a \rho$, such that $\pi a<_{\mathcal{R}} \pi$ and $a \rho<_{\mathcal{L}} \rho$.

We omit next lemma's easy proof, analog to that of [31, Lemma 2.1].

Lemma 4.2. Let $\bigvee$ be a pseudovariety of semigroups. Suppose that $(\pi, a, \rho)$ is a good factorization of an element of $\bar{\Omega}_{A} \mathrm{~V}$. Let $\pi^{\prime}$ be a suffix of $\pi$ and let $\rho^{\prime}$ be a prefix of $\rho$. Then $\left(\pi^{\prime}, a, \rho^{\prime}\right)$ is a good factorization.

In the next definition the hypothesis $\mathrm{V} \supseteq \mathrm{N}$ is required to ensure that the elements of $A^{+}$embed in $\bar{\Omega}_{A} \vee$ (as isolated points).

Definition 4.3. Let $\mathrm{V}$ be a semigroup pseudovariety containing $\mathrm{N}$. A +good factorization of $x \in \bar{\Omega}_{A} \mathrm{~V}$ is a triple $(\pi, u, \rho)$ in $\left(\bar{\Omega}_{A} \mathrm{~V}\right)^{1} \times A^{+} \times\left(\bar{\Omega}_{A} \mathrm{~V}\right)^{1}$, with $x=\pi u \rho$, such that $\pi \mathrm{i}_{1}(u)<_{\mathcal{R}} \pi$ and $\mathrm{t}_{1}(u) \rho<_{\mathcal{L}} \rho$.

Note that a good factorization is + -good factorization (assuming $\mathrm{V} \supseteq \mathrm{N}$ ).

Starting in the next lemma, we use the usual notation $B(\pi, \varepsilon)$ for the open ball of center $\pi$ and radius $\varepsilon$.

Lemma 4.4. Let $\mathrm{V}$ be a pseudovariety of semigroups containing N. Suppose that $\pi \in\left(\bar{\Omega}_{A} \mathrm{~V}\right)^{1}$ and $a \in A$ are such that $\pi a<_{\mathcal{R}} \pi$. Then, there is a positive integer $k_{0}$ such that, for every $k \geq k_{0}$, and for every $u \in A^{*}$, the set

$$
\left[B\left(\pi, \frac{1}{k}\right) \cap A^{*}\right] \cdot a u
$$

is a prefix code.

Proof. The proof reduces immediately to the case $u=1$ (cf. first two sentences in Remark 2.6). Let $J$ be the set of positive integers $k$ for which $\left[B\left(\pi, \frac{1}{k}\right) \cap A^{*}\right] \cdot a$ is not a prefix code. Suppose that $J$ is infinite. For each $k \in J$, we may consider distinct elements $w_{k}$ and $z_{k}$ of $B\left(\pi, \frac{1}{k}\right) \cap A^{*}$ such that $w_{k} a$ is a prefix of $z_{k} a$. For such elements, there is $t_{k} \in A^{*}$ with $z_{k}=w_{k} a t_{k}$. Note that the sequences $\left(z_{k}\right)_{k \in J}$ and $\left(w_{k}\right)_{k \in J}$ converge to $\pi$ and that $\left(t_{k}\right)_{k \in J}$ has some accumulation point $t$ in the compact space $\left(\bar{\Omega}_{A} \mathrm{~V}\right)^{1}$. Hence, we have the equality $\pi=\pi a t$, contradicting the hypothesis that $\pi a<_{\mathcal{R}} \pi$. To avoid the contradiction, the set $J$ must be finite.

In the following proofs, we shall frequently use, without reference, that if $\mathrm{V}$ is a semigroup pseudovariety closed under bideterministic product, then $\mathrm{V}$ contains N (cf. Example 3.14).

Corollary 4.5. Let $\mathrm{V}$ be a pseudovariety of semigroups closed under bideterministic product. Suppose that $\pi \in\left(\bar{\Omega}_{A} \mathrm{~V}\right)^{1}$ and $a \in A$ are such that 
$\pi a<_{\mathcal{R}} \pi$. Then, there is a positive integer $k_{0}$ such that, for every $k \geq k_{0}$, and for every $u \in A^{*}$, the set $B\left(\pi, \frac{1}{k}\right) \cdot$ au is clopen.

Proof. By Lemma 4.4, there is a positive integer $k_{0}$ such that for every $k \geq k_{0}$, the set $\left[B\left(\pi, \frac{1}{k}\right) \cap A^{*}\right] \cdot a$ is a prefix code. Hence, for $k \geq k_{0}$ and $u \in A^{*}$, the language $\left[B\left(\pi, \frac{1}{k}\right) \cap A^{*}\right] \cdot a \cdot u$ is a bideterministic product of $\mathrm{V}$-recognizable languages, and thus it is itself $\mathrm{V}$-recognizable by the hypothesis that $\mathrm{V}$ is closed under bideterministic product. Taking the topological closure in $\bar{\Omega}_{A} \mathrm{~V}$, we conclude that $B\left(\pi, \frac{1}{k}\right) \cdot a u$ is clopen.

Lemma 4.6. Let $\mathrm{V}$ be a pseudovariety of semigroups closed under bideterministic product. Suppose that $(\pi, u, \rho)$ is a -good factorization of $x \in$ $\bar{\Omega}_{A} \mathrm{~V}$. Let $a, b \in A, v, w \in A^{*}$ be such that $u=a v=w b$. Then $(\pi, a, v \rho)$ and $(\pi w, b, \rho)$ are good factorizations of $x$.

Proof. By symmetry, it suffices to show that $(\pi, a, v \rho)$ is a good factorization. We suppose that $\rho \neq 1$ and $v \neq 1$, as both the cases $\rho=1$ and $v=1$ are trivial. We only need to show av $<_{\mathcal{L}} v \rho$. Suppose on the contrary that av $\rho \mathcal{L} v \rho$. Then $v \rho=z a v \rho$ for some $z \in \bar{\Omega}_{A} \mathrm{~V}$. Let $\left(z_{n}\right)_{n}$ and $\left(\rho_{n}\right)_{n}$ be sequences of elements of $A^{+}$respectively converging to $z$ and $\rho$. Thanks to the dual of Corollary 4.5, there is a positive integer $k_{0}$ such that, for every $k \geq k_{0}$, the set $v \cdot B\left(\rho, \frac{1}{k}\right)$ is a clopen neighborhood of $v \rho$. Therefore, as $z_{n} a v \rho_{n}$ converges to $z a v \rho=v \rho$, we can build subsequences $\left(z_{n_{k}}\right)_{k \geq k_{0}}$ and $\left(\rho_{n_{k}}\right)_{k \geq k_{0}}$ such that $z_{n_{k}} a v \rho_{n_{k}} \in v \cdot B\left(\rho, \frac{1}{k}\right)$, with $\rho_{n_{k}} \in B\left(\rho, \frac{1}{k}\right)$, for every $k \geq k_{0}$. But then $z_{n_{k}} a v \rho_{n_{k}}$ is an element of the intersection

$$
A^{+} \cdot v\left[B\left(\rho, \frac{1}{k}\right) \cap A^{*}\right] \cap v\left[B\left(\rho, \frac{1}{k}\right) \cap A^{*}\right],
$$

for every $k \geq k_{0}$. This contradicts the dual of Lemma 4.4,

Proposition 4.7. Let $\mathrm{V}$ be a pseudovariety of semigroups closed under bideterministic product. Suppose that $(\pi, u, \rho)$ is a +-good factorization of an element $x$ of $\bar{\Omega}_{A} \mathrm{~V}$. For each positive integer $k$, consider the subset $L_{k}(\pi, u, \rho)$ of $\bar{\Omega}_{A} \mathrm{~V}$ defined by:

$$
L_{k}(\pi, u, \rho)=B\left(\pi, \frac{1}{k}\right) \cdot u \cdot B\left(\rho, \frac{1}{k}\right) .
$$

For all sufficiently large $k$, the set $L_{k}(\pi, u, \rho)$ is a clopen neighborhood of $x$.

Proof. Since $\bar{\Omega}_{A} \vee \backslash A^{+}$is an ideal of $\bar{\Omega}_{A} \vee$, we have

$$
L_{k}(\pi, u, \rho) \cap A^{+}=\left[B\left(\pi, \frac{1}{k}\right) \cap A^{*}\right] \cdot u \cdot\left[B\left(\rho, \frac{1}{k}\right) \cap A^{*}\right] .
$$

By Lemma 4.4, there is a positive integer $k_{1}$ such that, for every $k \geq k_{1}$, the set $\left[B\left(\pi, \frac{1}{k}\right) \cap A^{*}\right] \cdot \mathrm{i}_{1}(u)$ is a prefix code. By the dual of Lemma 4.4, there is a positive integer $k_{2}$, such that, for every $k \geq k_{1}$, the set $\mathrm{t}_{1}(u) \cdot\left[B\left(\pi, \frac{1}{k}\right) \cap A^{*}\right]$ 
is a suffix code. Therefore, for $k \geq \max \left\{k_{1}, k_{2}\right\}$, the product in the right side of (4.1) is $\mathrm{V}$-recognizable (cf. Remark 2.6), and so its closure in $\bar{\Omega}_{A} \mathrm{~V}$, the set $L_{k}(u, a, v)$, is open.

The proof of the following proposition is an adaptation of part of the proof of [8, Lemma 3.2], where a description of compact metric semigroups with open multiplication is given in terms of a property of sequences.

Proposition 4.8. Let $\mathrm{V}$ be a semigroup pseudovariety closed under bideterministic product. Suppose that $(\pi, u, \rho)$ is a +-good factorization of $x \in$ $\bar{\Omega}_{A} \mathrm{~V}$. Let $\left(x_{n}\right)_{n}$ be a sequence of elements of $\bar{\Omega}_{A} \mathrm{~V}$ converging to $x$. There are sequences $\left(\pi_{n}\right)_{n}$ and $\left(\rho_{n}\right)_{n}$ in $\left(\bar{\Omega}_{A} \mathrm{~V}\right)^{1}$, respectively converging to $\pi$ and $\rho$, such that $x_{n}=\pi_{n} u \rho_{n}$ for all sufficiently large $n$.

Proof. For each integer $k \geq 1$, consider the set $L_{k}(\pi, u, \rho)$ as in Proposition 4.7, and let $k_{0}$ be such that $L_{k}(\pi, u, \rho)$ is a clopen neighborhood of $x$ for every $k \geq k_{0}$ (such $k_{0}$ exists by Proposition 4.7). For each $k \geq k_{0}$, take $p_{k} \in \mathbb{Z}^{+}$such that $x_{n} \in L_{k}(\pi, u, \rho)$ when $n \geq p_{k}$. Let $\left(n_{k}\right)_{k \geq k_{0}}$ be the strictly increasing sequence defined by $n_{k_{0}}=p_{k_{0}}$ and $n_{k}=\max \left\{n_{k-1}+1, p_{k}\right\}$ for $k>k_{0}$. When $n_{k} \leq n<n_{k+1}$, take $\pi_{n} \in B\left(\pi, \frac{1}{k}\right)$ and $\rho_{n} \in B\left(\rho, \frac{1}{k}\right)$ such that $x_{n}=\pi_{n} u \rho_{n}$, which we can do as $x_{n} \in L_{k}(\pi, u, \rho)$. If $n<n_{k_{0}}$, take $\pi_{n}=\rho_{n}=1$. Clearly, $\left(\pi_{n}\right)_{n}$ and $\left(\rho_{n}\right)_{n}$ respectively converge to $\pi$ and $\rho$.

We are ready to prove the next theorem, a sort of generalization of the equidivisibility property, observed in [7], of the finitely generated free profinite semigroups over pseudovarieties closed under unambiguous product.

Theorem 4.9. Let $\mathrm{V}$ be a pseudovariety of semigroups closed under bideterministic product. Suppose that $(u, a, v)$ is a good factorization of an element $x$ of $\bar{\Omega}_{A} \mathrm{~V}$. Let $x=$ wbz be a factorization of $x$ such that $b \in A$. Then, at least one of the three following cases occurs:

(1) $u=w, a=b$ and $v=z$;

(2) $u=w b t$ and $z=$ tav for some $t \in\left(\bar{\Omega}_{A} \mathrm{~V}\right)^{1}$;

(3) $w=$ uat and $v=t b z$ for some $t \in\left(\bar{\Omega}_{A} \mathrm{~V}\right)^{1}$.

Proof. As $A^{*}$ is dense in $\left(\bar{\Omega}_{A} \mathrm{~V}\right)^{1}$, we may consider sequences $\left(w_{n}\right)_{n}$ and $\left(z_{n}\right)_{n}$ of elements of $A^{*}$ respectively converging to $w$ and $z$. Let $x_{n}=w_{n} b z_{n}$. Note that $\lim x_{n}=x$, and so, by Proposition 4.8, there are sequences $\left(u_{n}\right)_{n}$ and $\left(v_{n}\right)_{n}$ of elements of $A^{*}$, respectively converging to $u$ and $v$, and a positive integer $p$, such that $u_{n} a v_{n}=w_{n} b z_{n}, n \geq p$. Since the latter is an equality of words of $A^{*}$, for each $n \geq p$ one of the following three situations occurs:

(a) $u_{n}=w_{n}, a=b$ and $v_{n}=z_{n}$;

(b) $u_{n}=w_{n} b t_{n}$ and $z_{n}=t_{n} a v_{n}$ for some $t_{n} \in A^{*}$;

(c) $w_{n}=u_{n} a t_{n}$ and $v_{n}=t_{n} b z_{n}$ for some $t_{n} \in A^{*}$.

We let $J_{1}, J_{2}$ and $J_{3}$ be the sets of positive integers $n$ greater or equal than $p$ for which, respectively, situations (a), (b) and (c) occur. At least one of the three sets is infinite. Suppose that $J_{2}$ is infinite. For each $n \in J_{2}$, let $t_{n} \in A^{*}$ 
be as in (b), By compactness, the sequence $\left(t_{n}\right)_{n \in J_{2}}$ has some accumulation point $t$ in $\left(\bar{\Omega}_{A} \mathrm{~V}\right)^{1}$. Taking limits, we get $u=w b t$ and $z=t a v$, and so if $J_{2}$ is infinite then Case (2) holds. Arguing in a similar manner, we conclude that Case (3) holds if $J_{3}$ is infinite, and that Case (11) holds if $J_{1}$ is infinite.

\section{Pseudowords without good factorizations}

An analog of the next proposition, and of the corollary following it, is implicitly proved in [31] for good factorizations with respect to a homomorphism defined in a free monoid (cf. proof of [31, Theorem 2.6]).

Proposition 5.1. Let $\mathrm{V}$ be a pseudovariety of semigroups closed under bideterministic product. The set of elements of $\bar{\Omega}_{A} \mathrm{~V}$ without good factorizations is a closed subsemigroup of $\bar{\Omega}_{A} \mathrm{~V}$.

Proof. Denote by $S$ the set of elements of $\bar{\Omega}_{A} \vee$ without good factorizations.

Let $x, y \in S$. Suppose that $x y$ has some good factorization $(u, a, v)$. Take $b \in A$ and $z \in\left(\bar{\Omega}_{A} \mathrm{~V}\right)^{1}$ such that $y=b z$. Applying Theorem 4.9 to $u \cdot a \cdot v=x \cdot b \cdot z$, we conclude that one of the following occurs:

(1) $u=x, a=b$ and $v=z$;

(2) $u=x b t$ and $z=t a v$ for some $t \in\left(\bar{\Omega}_{A} \mathrm{~V}\right)^{1}$;

(3) $x=u a t$ and $v=t b z$ for some $t \in\left(\bar{\Omega}_{A} \mathrm{~V}\right)^{1}$.

In the first case, as $(u, a, v)$ is a good factorization, so is $(1, a, v)=(1, b, z)$. But $b z=y$ has no good factorizations, by hypothesis, and so the first case does not hold. If we are in the second case, then, as $b t$ is a suffix of $u$, we deduce from Lemma 4.2 that $(b t, a, v)$ is a good factorization of $b z=y$, contradicting the hypothesis that $y$ has no good factorizations. Similarly, the third case is in contradiction with $x$ not having good factorizations. Therefore, $x y$ has no good factorization, and $S$ is a subsemigroup of $\bar{\Omega}_{A} \mathrm{~V}$.

Finally, let $\left(x_{n}\right)_{n}$ be a sequence of elements of $S$ converging in $\bar{\Omega}_{A} \mathrm{~V}$ to $x \in$ $\bar{\Omega}_{A} \mathrm{~V}$. Suppose that $x \notin S$. We may then consider a good factorization $(u, a, v)$ of $x$. By Proposition 4.8, there are sequences $\left(u_{n}\right)_{n}$ and $\left(v_{n}\right)_{n}$ of elements of $\left(\bar{\Omega}_{A} \mathrm{~V}\right)^{1}$, respectively converging to $u$ and $v$, and there is $p$ such that $x_{n}=u_{n} a v_{n}$ for all $n \geq p$. Consider the sets

$$
J_{1}=\left\{n \geq p \mid u_{n} a \mathcal{R} u_{n}\right\} \quad \text { and } \quad J_{2}=\left\{n \geq p \mid a v_{n} \mathcal{L} v_{n}\right\} .
$$

Since $x_{n} \in S$, every integer $n$ greater or equal to $p$ belongs to $J_{1} \cup J_{2}$, and so at least one of the sets $J_{1}$ and $J_{2}$ is infinite. Suppose that $J_{1}$ is infinite. Since $\mathcal{R}$ is a closed relation in $\left(\bar{\Omega}_{A} \mathrm{~V}\right)^{1}$, taking limits we get $u a \mathcal{R} u$, contradicting that $(u, a, v)$ is a good factorization. Similarly, a contradiction arises if $J_{2}$ is infinite. Therefore, $x \in S$ and so $S$ is closed.

Corollary 5.2. Let $\mathrm{V}$ be a pseudovariety of semigroups closed under bideterministic product. If $\pi$ is a product of regular elements of $\bar{\Omega}_{A} \mathrm{~V}$, then $\pi$ has no good factorization.

Proof. By Proposition [5.1, it suffices to show that an arbitrary regular element $\pi$ of $\bar{\Omega}_{A} \vee$ has no good factorizations. Take $s \in \bar{\Omega}_{A} \vee$ such that $\pi=\pi s \pi$. 
Suppose there is a good factorization $(x, a, y)$ of $\pi$. As $\pi=x \cdot a \cdot y s \pi$, by Theorem 4.9 one of three cases holds: $y=y s \pi$, or $x a$ is a prefix of $x$, or ays $\pi$ is a suffix of $y$. The second case immediately contradicts $(x, a, y)$ being a good factorization. And since $a y$ is a suffix of $\pi$, in the first and third cases we get $y \mathcal{L}$ ay, also a contradiction. Hence, $\pi$ has no good factorization.

The next lemma and the theorem that follows it are proved in 31] for the corresponding monoid versions. We prove them in the semigroup versions with a somewhat different approach: we use pseudowords. For a semigroup $S$, we let $\operatorname{Reg}(S)$ be the set of regular elements of $S$, and let $\langle X\rangle$ be the subsemigroup of $S$ generated by a nonempty subset $X$ of $S$.

Lemma 5.3. Let $\varphi: A^{+} \rightarrow S$ be a homomorphism onto a finite semigroup, and let $u \in A^{+}$be such that $\varphi_{\mathrm{bd}}(u)$ is a product of regular elements of $S_{\varphi}$. Then $u$ has no good factorizations with respect to $\varphi$.

Proof. Consider the unique continuous homomorphism $\widehat{\varphi_{\mathrm{bd}}}: \bar{\Omega}_{A} \mathrm{~S} \rightarrow S_{\varphi}$ extending $\varphi_{\text {bd }}$. Every regular element of $S_{\varphi}$ is the image by $\widehat{\varphi_{\mathrm{bd}}}$ of a regular element of $\bar{\Omega}_{A} \mathrm{~S}$, and so we may take $w$ in $\left\langle\operatorname{Reg}\left(\bar{\Omega}_{A} \mathrm{~S}\right)\right\rangle$ with $\varphi_{\mathrm{bd}}(u)=\widehat{\varphi_{\mathrm{bd}}}(w)$. Let $\left(w_{n}\right)_{n}$ be a sequence of words converging to $w$. Take the set $J$ of positive integers $n$ such that $w_{n}$ has a good factorization $\left(x_{n}, a_{n}, y_{n}\right)$ with respect to $\varphi$. Suppose that $J$ is infinite. Let $(x, a, y)$ be an accumulation point of $\left(x_{n}, a_{n}, y_{n}\right)_{n \in J}$. Then $w=x a y$, and for every $n$ in an infinite subset of $J$, one has $\varphi\left(x_{n}\right)=\widehat{\varphi}(x), a_{n}=a$ and $\varphi\left(y_{n}\right)=\widehat{\varphi}(y)$. Therefore, $\widehat{\varphi}(x)<_{\mathcal{R}} \widehat{\varphi}(x a)$ and $\widehat{\varphi}(y)<_{\mathcal{L}} \widehat{\varphi}(a y)$ hold, thus $x<_{\mathcal{R}} x a$ and $y<_{\mathcal{L}} a y$. Hence, $(x, a, y)$ is a good factorization of $w$. But this contradicts Corollary 5.2, and so $J$ must be finite. As $\widehat{\varphi_{\mathrm{bd}}}(w)=\varphi_{\mathrm{bd}}\left(w_{n}\right)$ for all large enough $n$, we conclude that $\varphi_{\mathrm{bd}}(u)=\varphi_{\mathrm{bd}}(v)$ for some $v \in A^{+}$without good factorizations with respect to $\varphi$. By the definition of the congruence $\sim_{\varphi}$, it follows that $u$ has no good factorizations with respect to $\varphi$.

Theorem 5.4. Let $\varphi: A^{+} \rightarrow S$ be a homomorphism onto a finite semigroup. Then $p_{\varphi}: S_{\varphi} \rightarrow S$ restricts to an isomorphism $\left\langle\operatorname{Reg}\left(S_{\varphi}\right)\right\rangle \rightarrow\langle\operatorname{Reg}(S)\rangle$.

Proof. Since $S_{\varphi}$ is finite, we have $p_{\varphi}\left(\left\langle\operatorname{Reg}\left(S_{\varphi}\right)\right\rangle\right)=\langle\operatorname{Reg}(S)\rangle$, so it remains to show the restriction is one-to-one. Take $s, t \in\left\langle\operatorname{Reg}\left(S_{\varphi}\right)\right\rangle$. Let $u, v \in A^{+}$be such that $s=\varphi_{\mathrm{bd}}(u)$ and $t=\varphi_{\mathrm{bd}}(v)$. By Lemma 5.3, both $u$ and $v$ have no good factorizations with respect to $\varphi$. Therefore, we have $\varphi_{\mathrm{bd}}(u)=\varphi_{\mathrm{bd}}(v)$ if and only if $\varphi(u)=\varphi(v)$, that is, $s=t$ if and only if $p_{\varphi}(s)=p_{\varphi}(t)$.

For the sake of conciseness, say that a pseudoword $\pi \in \bar{\Omega}_{A} \mathrm{~S}$ is $\mathrm{V}$-regular when $[\pi]_{\vee}$ is regular, and $\pi$ is $\vee$-multiregular if $[\pi]_{\vee}$ is a finite product of V-regular pseudowords (actually, one may drop the finiteness assumption in subsequent results, but the assumption is nevertheless included because of the examples we have in mind).

The following result is a sufficient condition to "climb up" a pseudoidentity from $\mathrm{V}$ to $\mathrm{V}_{\mathrm{bd}}$. 
Proposition 5.5. Let $\mathrm{V}$ be a pseudovariety of semigroups. If $\pi, \rho \in \bar{\Omega}_{X} \mathrm{~S}$ are $\mathrm{V}_{\mathrm{bd}}$-multiregulars, then $\mathrm{V} \models \pi=\rho$ implies $\mathrm{V}_{\mathrm{bd}} \models \pi=\rho$.

Proof. Let $\varphi: A^{+} \rightarrow S$ be a homomorphism onto a semigroup $S$ of V. Take an arbitrary homomorphism $\psi: X^{+} \rightarrow S_{\varphi}$. Let us show that $\widehat{\psi}(\pi)=\widehat{\psi}(\rho)$.

Since $\widehat{\varphi \mathrm{bd}}$ is onto, by the freeness of $\bar{\Omega}_{X} \mathrm{~S}$ there is a continuous homomorphism $\zeta: \bar{\Omega}_{X} S \rightarrow \bar{\Omega}_{A} \mathrm{~S}$ such that $\widehat{\psi}=\widehat{\varphi \mathrm{bd}} \circ \zeta$. As $S \models \pi=\rho$, we have

$$
\widehat{\varphi}(\zeta(\pi))=\widehat{\varphi}(\zeta(\rho)) .
$$

As $S_{\varphi} \in \mathrm{V}_{\mathrm{bd}}$, there is a continuous homomorphism $\beta: \bar{\Omega}_{A} \mathrm{~V}_{\mathrm{bd}} \rightarrow S_{\varphi}$ such that

$$
\widehat{\varphi \mathrm{bd}}=\beta \circ p_{\mathrm{V}_{\mathrm{bd}}} .
$$

From the hypothesis that $\pi$ and $\rho$ are $\mathrm{V}_{\mathrm{bd}}$-multiregulars we get that the pseudowords $\zeta(\pi)$ and $\zeta(\rho)$ are also $\mathrm{V}_{\mathrm{bd}}$-multiregulars, and so, in view of (5.2), we conclude that $\widehat{\varphi_{\mathrm{bd}}}(\zeta(\pi))$ and $\widehat{\varphi_{\mathrm{bd}}}(\zeta(\rho))$ are both products of regular elements of $S_{\varphi}$. Then, applying Theorem 5.4, we obtain from equality (5.1) the equality $\widehat{\varphi_{\mathrm{bd}}}(\zeta(\pi))=\widehat{\varphi_{\mathrm{bd}}}(\zeta(\rho))$, that is $\widehat{\psi}(\pi)=\widehat{\psi}(\rho)$. Since $\psi$ is an arbitrary homomorphism from $X^{+}$into $S_{\varphi}$, we conclude that $S_{\varphi} \models \pi=\rho$. This shows that $\mathrm{V}_{\mathrm{bd}}=\pi=\rho$.

The characterization of $\bar{V}$ observed in Remark 3.6 (more precisely, the semigroup pseudovariety version of Remark 3.6) and Proposition 5.5 allow us to deduce the following, with a straightforward inductive argument.

Corollary 5.6. Let $\vee$ be a pseudovariety of semigroups. If $\pi, \rho \in \bar{\Omega}_{X} \mathrm{~S}$ are $\overline{\mathrm{V}}$-multiregulars, then $\mathrm{V} \models \pi=\rho$ implies $\overline{\mathrm{V}} \models \pi=\rho$.

Definition 5.7. Let $\mathrm{V}$ and $\mathrm{W}$ be semigroup pseudovarieties with $\mathrm{V} \subseteq \mathrm{W}$. Say that $\mathrm{V}$ is multiregularly based in $\mathrm{W}$ if it has a basis $\Sigma$ of pseudoidentities such that, for every pseudoidentity $(\pi=\rho)$ in $\Sigma$, both $\pi$ and $\rho$ are Wmultiregular pseudowords. If $\mathrm{W}=\mathrm{S}$, then we just say that $\mathrm{V}$ is multiregularly based.

Proposition 5.8. Let $\mathrm{V}$ and $\mathrm{W}$ be pseudovarieties of semigroups with $\mathrm{V} \subseteq$ $\mathrm{W}$ and $\mathrm{V}$ is multiregularly based in $\mathrm{W}$. If $\mathrm{W}$ is closed under bideterministic product, then so is $\mathrm{V}$.

Proof. Let $\Sigma$ be a basis for $\mathrm{V}$ such that, for every pseudoidentity $(\pi=\rho)$ in $\Sigma$, both $\pi$ and $\rho$ are W-multiregulars. Fix an element $(\pi=\rho)$ of $\Sigma$. Since $\mathrm{V} \subseteq \mathrm{W}$ and $\mathrm{W}$ is closed under bideterministic product, the inclusion $\mathrm{V}_{\mathrm{bd}} \subseteq \mathrm{W}$ holds. It then follows from Proposition 5.5 that $\mathrm{V}_{\mathrm{bd}} \models \pi=\rho$. This shows that $\mathrm{V}_{\mathrm{bd}} \subseteq \mathrm{V}$, that is, $\mathrm{V}$ is closed under bideterministic product.

Example 5.9. The pseudovarieties DS $=\llbracket\left((x y)^{\omega}(y x)^{\omega}(x y)^{\omega}\right)^{\omega}=(x y)^{\omega} \rrbracket$ and $\mathbf{J}=\llbracket(x y)^{\omega}=(y x)^{\omega}, x^{\omega+1}=x^{\omega} \rrbracket$ are pseudovarieties of semigroups closed under bideterministic product, in view of Proposition 5.8. 
Corollary 5.10. If $\mathrm{V}$ is a pseudovariety of semigroups multiregularly based then the pseudovariety $\mathcal{L} \mathrm{V}$ is closed under bideterministic product.

Proof. Suppose that $\mathrm{V}=\llbracket \Sigma \rrbracket$, where $\Sigma$ is a set of pseudoidentities. For each pseudoidentity $(u=v) \in \Sigma$, consider an alphabet $A$ that contains $c(u) \cup c(v)$ and a letter $z \notin c(u) \cup c(v)$. Let $\varphi_{u, v}$ be the unique continuous endomorphism of $\bar{\Omega}_{A} \mathrm{~S}$ such that $\varphi(x)=z^{\omega} x z^{\omega}$ for every letter $x$ of $A$. Then the equality $\mathcal{L} \mathrm{V}=\llbracket \varphi_{u, v}(u)=\varphi_{u, v}(v) \mid(u=v) \in \Sigma \rrbracket$ holds. Clearly, if $u$ and $v$ are multiregulars, then the same happens with $\varphi_{u, v}(u)$ and $\varphi_{u, v}(v)$. Hence, if $\mathrm{V}$ is multiregularly based, then $\mathcal{L} \mathrm{V}$ is multiregularly based and, by Proposition (5.8), LV is closed under bideterministic product.

We close this section applying Corollary 5.2 in the proof of the following technical lemma, to be used later on.

Lemma 5.11. Let $\mathrm{V}$ be a pseudovariety of semigroups closed under bideterministic product. Consider an element $x$ of $\bar{\Omega}_{A} \vee$ such that $x \leq_{\mathcal{R}} y$ for some regular element $y$ of $\bar{\Omega}_{A} \mathrm{~V}$. Then, there is not a good factorization $(u, a, \pi)$ of $x$ such that $u \in A^{*}$.

Proof. Suppose, on the contrary, that there is a good factorization $(u, a, \pi)$ of $x$ such that $u \in A^{*}$. Let $x=y z$, with $z \in\left(\bar{\Omega}_{A} \mathrm{~V}\right)^{1}$. Since $y$ is regular, it has a factorization $y=y_{0} \cdot b \cdot y_{1}$, such that $y_{0} \in A^{*}, b \in A, y_{1} \in \bar{\Omega}_{A} \vee$ and $\left|y_{0}\right|=|u|$. Applying Theorem 4.9 to compare the factorizations $(u, a, \pi)$ and $\left(y_{0}, b, y_{1} z\right)$ of $x$, and since $u$ and $y_{0}$ are finite words of the same length, we conclude that $u=y_{0}, a=b$ and $\pi=y_{1} z$. As the triple $\left(y_{0}, b, y_{1} z\right)$ is then a good factorization of $x$, we may apply Lemma4.2 to conclude that the triple $\left(y_{0}, a, y_{1}\right)$ is a good factorization of $y$. But this contradicts Corollary 5.2 .

\section{ORGANIZED FACTORIZATIONS}

In this section, we quickly review the factorizations of pseudowords as products of words and regular elements over $\mathrm{J}$, and then proceed to an abstraction of that property. First, it is convenient to recall the following properties, going back to [14]. We give [1, Chapter 8] as reference.

Proposition 6.1. Let $\pi, \rho \in \bar{\Omega}_{A} \mathrm{~S}$. The following properties hold:

(1) $\pi$ is J-regular if and only if $\pi$ is DS-regular;

(2) if $\pi$ and $\rho$ are $\mathrm{J}$-regular, then $\mathrm{J} \models \pi=\rho$ if and only if $c(\pi)=c(\rho)$;

(3) for every pseudovariety of semigroups $\mathrm{V}$ such that $\mathrm{SI} \subseteq \mathrm{V} \subseteq \mathrm{DS}$, if $\pi$ is $\mathrm{V}$-regular, then $\mathrm{V} \models \pi \rho \mathcal{R} \pi$ if and only if $c(\rho) \subseteq c(\pi)$.

Definition 6.2. A factorization $\pi=u_{0} \cdot \pi_{1} \cdot u_{1} \cdot \pi_{2} \cdots \pi_{n-1} \cdot u_{n-1} \cdot \pi_{n} \cdot u_{n}$ of an element $\pi$ of $\bar{\Omega}_{A} S$ is J-reduced if the next four conditions are satisfied:

(1) $u_{i} \in A^{*}$ for every $i \in\{0,1, \ldots, n\}$;

(2) $\pi_{i}$ is J-regular for every $i \in\{1, \ldots, n\}$;

(3) if $u_{i}=1$ and $1 \leq i \leq n-1$, then $c\left(\pi_{i}\right)$ and $c\left(\pi_{i+1}\right)$ are incomparable;

(4) $\mathrm{t}_{1}\left(u_{i-1}\right) \notin c\left(\pi_{i}\right)$ and $\mathrm{i}_{1}\left(u_{i}\right) \notin c\left(\pi_{i}\right)$, for every $i \in\{1, \ldots, n\}$. 
If, moreover, the fifth condition $u_{0}=u_{1}=\ldots=u_{n-1}=u_{n}=1$ is also satisfied, then we say that $\pi=\pi_{1} \pi_{2} \cdots \pi_{n-1} \pi_{n}$ is a J-reduced multiregular element of $\bar{\Omega}_{A} \mathrm{~S}$.

Lemma 6.3. Suppose that $\pi \in \bar{\Omega}_{A} \mathrm{~S}$ is a product of $n$ pseudowords that are $\mathrm{J}$-regular. Then $\pi$ factorizes as a J-reduced multiregular pseudoword $\pi_{1} \ldots \pi_{k}$ for some $k \leq n$.

Proof. Let $\pi=\pi_{1} \cdots \pi_{n}$ be a factorization into J-regular pseudowords. We show the lemma by induction on $n$. The base case is trivial. Suppose the lemma holds for smaller values of $n$. If $c\left(\pi_{i}\right)$ and $c\left(\pi_{i+1}\right)$ are incomparable for each $i \in\{1, \ldots, n-1\}$, then the factorization is already Jreduced. If, on the contrary, $c\left(\pi_{j}\right)$ and $c\left(\pi_{j+1}\right)$ are comparable for some $j$, then $\pi_{j}^{\prime}=\pi_{j} \pi_{j+1}$ is a J-regular pseudoword (cf. Proposition 6.1)(3) ) and $\pi=\pi_{1} \cdots \pi_{j-1} \pi_{j}^{\prime} \pi_{j+2} \cdots \pi_{n}$ is a factorization of $\pi$ into less than $n$ J-regular factors. We may then apply the induction hypothesis.

As supporting references for the next theorem, we give [4, Section 4] and [1, Theorem 8.1.11].

Theorem 6.4. Every element of $\bar{\Omega}_{A} \mathrm{~S}$ has a J-reduced factorization, and each $\mathrm{J}$-reduced factorization is unique modulo $\mathrm{J}$, that is, if

$$
\begin{aligned}
& \pi=u_{0} \cdot \pi_{1} \cdot u_{1} \cdot \pi_{2} \cdots \pi_{n-1} \cdot u_{n-1} \cdot \pi_{n} \cdot u_{n}, \\
& \rho=v_{0} \cdot \rho_{1} \cdot v_{1} \cdot \rho_{2} \cdots \rho_{m-1} \cdot v_{m-1} \cdot \rho_{m} \cdot v_{m},
\end{aligned}
$$

are $\mathrm{J}$-reduced factorizations such that $\mathrm{J} \models \pi=\rho$, then $m=n, u_{i}=v_{i}$ and $\mathrm{J} \models \pi_{j}=\rho_{j}$ for every $i \in\{0,1, \ldots, n\}$ and $j \in\{1, \ldots, n\}$.

The following interesting observation will be used later on.

Lemma 6.5. Let $\mathrm{V}$ be a semigroup pseudovariety in the interval [J, DS]. Suppose that $\pi=\pi_{1} \cdots \pi_{n} \in \bar{\Omega}_{A} \mathrm{~S}$ is a J-reduced multiregular pseudoword, and let $\rho \in \bar{\Omega}_{A} \mathrm{~S}$. Then $\mathrm{V} \models \pi \rho \mathcal{R} \pi$ if and only if $c(\rho) \subseteq c\left(\pi_{n}\right)$.

Proof. As $\mathrm{V} \models \pi_{n} \rho \mathcal{R} \pi_{n}$ implies $\mathrm{V} \models \pi \rho \mathcal{R} \pi$, the "if" part is immediate in view of Proposition 6.1. Conversely, suppose that $\mathrm{V} \models \pi \rho \mathcal{R} \pi$, and let $x$ be such that $\mathrm{V} \models \pi=\pi \rho x$. We then have $\mathrm{V} \models \pi_{1} \cdots \pi_{n}=\pi_{1} \cdots \pi_{n}(\rho x)^{\omega}$. By the uniqueness of J-reduced factorizations (Theorem 6.4), the sets $c\left(\pi_{n}\right)$ and $c\left((\rho x)^{\omega}\right)$ must be comparable, and so $\pi_{n}^{\prime}=\pi_{n}(\rho x)^{\omega}$ is $\mathrm{V}$-regular. Again by the uniqueness of J-reduced factorizations and by Lemma 6.3, the factorization $\pi_{1} \cdots \pi_{n-1} \pi_{n}^{\prime}$ must be J-reduced, and moreover $\mathrm{J} \models \pi_{n}=\pi_{n}^{\prime}$. In particular, we have $c(\rho) \subseteq c\left(\pi_{n}\right)$.

Next is an abstraction of some features of being J-reduced.

Definition 6.6. Let us consider a factorization of $\pi \in \bar{\Omega}_{A} S$ of the form

$$
\pi=u_{0} \cdot \pi_{1} \cdot u_{1} \cdot \pi_{2} \cdots \pi_{n-1} \cdot u_{n-1} \cdot \pi_{n} \cdot u_{n},
$$

for some $n \geq 0$, such that $u_{0}, u_{n} \in A^{*}, u_{i} \in A^{+}$when $1 \leq i \leq n-1$, and $\pi_{i} \notin A^{+}$when $1 \leq i \leq n$. 
Let $\mathrm{V}$ be a semigroup pseudovariety. The factorization (6.1) is multiregularly organized in $\mathrm{V}$ if the pseudowords $\pi_{i}, 1 \leq i \leq n$, are $\mathrm{V}$-multiregular. The same factorization (6.1) is organized with short $\mathrm{V}$-breaks if the following conditions hold:

(SB.1) $\left[\pi_{i} \mathrm{i}_{1}\left(u_{i}\right)\right]_{\vee}<_{\mathcal{R}}\left[\pi_{i}\right] \vee$ when $1 \leq i \leq n-1$;

(SB.2) $\left[\mathrm{t}_{1}\left(u_{i}\right) \pi_{i+1}\right]_{\vee}<_{\mathcal{L}}\left[\pi_{i+1}\right] \vee$ when $1 \leq i \leq n-1$;

(SB.3) if $u_{0} \neq 1$ then $\left[\mathrm{t}_{1}\left(u_{0}\right) \pi_{1}\right]_{\vee}<_{\mathcal{L}}\left[\pi_{1}\right]_{\mathrm{V}}$;

(SB.4) if $u_{n} \neq 1$ then $\left[\pi_{n} \mathrm{i}_{1}\left(u_{n}\right)\right]_{\mathrm{V}}<_{\mathcal{R}}\left[\pi_{n}\right]_{\mathrm{V}}$.

Finally, the factorization (6.1) is organized with long $\mathrm{V}$-breaks if the following conditions hold:

$$
\begin{aligned}
& \text { (LB.1) } {\left[u_{0} \pi_{1} u_{1} \pi_{2} \cdots \pi_{i-1} u_{i-1} \pi_{i} \mathrm{i}_{1}\left(u_{i}\right)\right]_{\mathrm{V}}<_{\mathcal{R}}\left[u_{0} \pi_{1} u_{1} \pi_{2} \cdots \pi_{i-1} u_{i-1} \pi_{i}\right]_{\mathrm{V}} \text { for each } } \\
& i \in\{1, \ldots, n-1\} ; \\
& \text { (LB.2) } {\left[\mathrm{t}_{1}\left(u_{i}\right) \pi_{i+1} u_{i+1} \pi_{i+2} \cdots u_{n-1} \pi_{n} u_{n}\right]_{\mathrm{V}}<_{\mathcal{L}}\left[\pi_{i+1} u_{i+1} \pi_{i+2} \cdots u_{n-1} \pi_{n} u_{n}\right]_{\mathrm{V}} } \\
& \text { for each } i \in\{1, \ldots, n-1\} ; \\
& \text { (LB.3) if } u_{0} \neq 1 \text { then }\left[\mathrm{t}_{1}\left(u_{0}\right) \pi_{1} u_{1} \pi_{2} \cdots u_{n-1} \pi_{n} u_{n}\right]_{\mathrm{V}}<_{\mathcal{L}}\left[\pi_{1} u_{1} \pi_{2} \cdots u_{n-1} \pi_{n} u_{n}\right]_{\mathrm{V}} ; \\
& \text { (LB.4) if } u_{n} \neq 1 \text { then }\left[u_{0} \pi_{1} u_{1} \pi_{2} \cdots u_{n-1} \pi_{n} \mathrm{i}_{1}\left(u_{n}\right)\right]_{\mathrm{V}}<_{\mathcal{R}}\left[u_{0} \pi_{1} u_{1} \pi_{2} \cdots u_{n-1} \pi_{n}\right]_{\mathrm{V}} .
\end{aligned}
$$

Remark 6.7. A factorization (6.1) that is organized with long $V$-breaks is a factorization that is organized with short V-breaks.

Definition 6.8. A semigroup pseudovariety $W$ is organizing if, for every alphabet $A$, every $\pi \in \bar{\Omega}_{A} S$ is multiregularly organized in W.

Example 6.9. The pseudovarieties DS and DS $\cap$ ECom are organizing pseudovarieties, the former by Theorem 6.4 (and Proposition 6.1(1)), the latter by an analog result of Almeida and Weil (Proposition 3.7 in [10]).

Proposition 6.10. Let $\mathrm{V}$ be a pseudovariety of semigroups. Suppose that $\pi$ is an element of $\bar{\Omega}_{A} \mathrm{~S}$ with a factorization multiregularly organized in a semigroup pseudovariety W. Then there are $\pi^{\prime} \in \bar{\Omega}_{A} S$ and a set $\Upsilon_{\pi}$ of pseudoidentities over $A$ satisfying the following conditions:

(1) $\vee \subseteq \llbracket \Upsilon_{\pi} \rrbracket \subseteq \llbracket \pi=\pi^{\prime} \rrbracket$;

(2) $\pi^{\prime}$ has a factorization multiregularly organized in $\mathrm{W}$ and with short V-breaks;

(3) if $(\varpi=\varrho)$ belongs to $\Upsilon_{\pi}$, then $\varpi$ and $\varrho$ are $\mathrm{W}$-multiregulars.

Proof. By hypothesis, there is a factorization

$$
\pi=u_{0} \cdot \pi_{1} \cdot u_{1} \cdot \pi_{2} \cdots \pi_{n-1} \cdot u_{n-1} \cdot \pi_{n} \cdot u_{n}
$$

which is multiregularly organized in W. Let $s=\sum_{i=0}^{n}\left|u_{i}\right|$ and $\sigma=n+s$. We show the proposition by induction on $\sigma \geq 0$. If $n=0$ or $s=0$ (the latter implies $n=1$ ), then just take $\pi^{\prime}=\pi$ and $\Upsilon_{\pi}=\emptyset$. In particular, this shows the initial step of the induction.

Suppose the proposition holds for smaller values of $\sigma$ and that $n>0$ and $s>0$. Let $J$ be the set of integers $i$ in $\{1, \ldots, n\}$ such that $\left[\pi_{i} \mathrm{i}_{1}\left(u_{i}\right)\right] \vee \mathcal{R}\left[\pi_{i}\right] \vee$ and $u_{i} \neq 1$, or such that $\left[\mathrm{t}_{1}\left(u_{i-1}\right) \pi_{i}\right] \vee \mathcal{L}\left[\pi_{i}\right] \vee$ and $u_{i-1} \neq 1$. In fact, one always has $u_{i} \neq 1$, except, perhaps, when $i=0$ or $i=n$. If $J=\emptyset$, then 
we just take $\pi^{\prime}=\pi$ and $\Upsilon_{\pi}=\emptyset$. Suppose that $J \neq \emptyset$, and let $j \in J$. Without loss of generality, we assume that $\left[\pi_{j} \mathrm{i}_{1}\left(u_{j}\right)\right] \vee \mathcal{R}\left[\pi_{j}\right] \vee$ and $u_{j} \neq 1$. Let $a=\mathrm{i}_{1}\left(u_{j}\right)$, and let $v \in A^{*}$ be such that $u_{j}=a v$. Then, there is $x \in \bar{\Omega}_{A} \mathrm{~S}$ such that

$$
\left[\pi_{j}\right] \mathrm{V}=\left[\pi_{j} a x\right]_{\mathrm{V}}=\left[\pi_{j}(a x)^{\omega}\right]_{\mathrm{V}}
$$

Let $\rho=\pi_{j}(a x)^{\omega} a$. Since $(a x)^{\omega} a$ is a regular element of $\bar{\Omega}_{A} S$ and $\left[\pi_{j}\right]_{\mathrm{W}}$ is $\mathrm{W}$-multiregular, we know that $[\rho]_{\mathrm{W}}$ is also $\mathrm{W}$-multiregular. Consider the pseudoword $\bar{\pi}$ with factorization

$$
\bar{\pi}=u_{0} \cdot \pi_{1} \cdot u_{1} \cdot \pi_{2} \cdots \pi_{j-1} \cdot u_{j-1} \cdot \rho \cdot v \cdot \pi_{j+1} \cdots \pi_{n-1} \cdot u_{n-1} \cdot \pi_{n} \cdot u_{n} .
$$

If $v \neq 1$, then this factorization is multiregularly organized in W. If $v=1$, then we also obtain a factorization of $\bar{\pi}$ which is multiregularly organized in W by "gluing" $\rho$ and $\pi_{j+1}$. In any case, we produce a factorization of $\bar{\pi}$ which is multiregularly organized in $\mathrm{W}$ and with smaller value for $\sigma$ than that we had in $\pi$. We may therefore apply the induction hypothesis to obtain a pseudoword $\pi^{\prime} \in \bar{\Omega}_{A} S$ and a set $\Upsilon_{\bar{\pi}}$ of pseudoidentities over $A$ satisfying the following conditions:

(1) $\vee \subseteq \llbracket \Upsilon_{\bar{\pi}} \rrbracket \subseteq \llbracket \bar{\pi}=\pi^{\prime} \rrbracket ;$

(2) $\pi^{\prime}$ has a factorization multiregularly organized in $W$ and with short V-breaks;

(3) if $(\varpi=\varrho)$ belongs to $\Upsilon_{\bar{\pi}}$, then $\varpi$ and $\varrho$ are $W$-multiregulars.

Let $\Upsilon_{\pi}=\Upsilon_{\bar{\pi}} \cup\left\{\left(\pi_{j}=\pi_{j}(a x)^{\omega}\right)\right\}$. By (6.2), we know that $\mathrm{V} \subseteq \llbracket \Upsilon_{\pi} \rrbracket$. Moreover, as $\left[\pi_{j}\right]_{\mathrm{W}}$ and $\left[\pi_{j}(a x)^{\omega}\right]_{\mathrm{W}}$ are both $\mathrm{W}$-multiregulars, we immediately get that Condition (3) in the statement of the proposition holds for $\Upsilon_{\pi}$. Consider a semigroup $S$ in $\llbracket \Upsilon_{\pi} \rrbracket$. Then $S$ belongs to $\llbracket \Upsilon_{\bar{\pi}} \rrbracket$, whence

$$
S \models \bar{\pi}=\pi^{\prime} .
$$

On the other hand, we also have $S \models \pi_{j}=\pi_{j}(a x)^{\omega}$, which implies that

$$
S \models \rho \cdot v=\pi_{j}(a x)^{\omega} a v=\pi_{j} u_{j} .
$$

We immediately conclude that $S \models \pi=\bar{\pi}=\pi^{\prime}$. We have therefore showed that $\llbracket \Upsilon_{\pi} \rrbracket \subseteq \llbracket \pi=\pi^{\prime} \rrbracket$, concluding the inductive step of the proof.

Corollary 6.11. Let $\mathrm{V}$ and $\mathrm{W}$ be pseudovarieties of semigroups, with $\mathrm{W}$ being an organizing pseudovariety. Then $\mathrm{V}$ has a basis of pseudoidentities with factorizations multiregularly organized in $\mathrm{W}$ and with short $\mathrm{V}$-breaks.

Proof. Let $\Sigma$ be a basis of pseudoidentities for $\mathrm{V}$. For each $(\pi=\rho) \in \Sigma$, let

$$
\Gamma_{(\pi=\rho)}=\Upsilon_{\pi} \cup \Upsilon_{\rho} \cup\left\{\left(\pi^{\prime}=\rho^{\prime}\right)\right\},
$$

where we follow the definition included in Proposition 6.10, Consider the union $\Gamma=\bigcup_{(\pi=\rho) \in \Sigma} \Gamma_{(\pi=\rho)}$. It suffices to show that $\mathrm{V}=\llbracket \Gamma \rrbracket$.

Suppose that $S \in \mathrm{V}$. For each $\pi$, one has $S \models \Upsilon_{\pi}$ and $S \models \pi=\pi^{\prime}$. In particular, $S \models \pi=\rho$ implies $S \models \pi^{\prime}=\rho^{\prime}$. We conclude that if $(\pi=\rho) \in \Sigma$, then $S \models \Gamma_{(\pi=\rho)}$ holds. This establishes the inclusion $\vee \subseteq \llbracket \Gamma \rrbracket$. 
Conversely, let $S \in \llbracket \Gamma \rrbracket$. Take $(\pi=\rho) \in \Sigma$. Because $S \models \Upsilon_{\pi}$, we have $S \models \pi=\pi^{\prime}$. Similarly, $S \models \rho=\rho^{\prime}$ holds. But $S \in \llbracket \Gamma \rrbracket$ also implies $S \models$ $\pi^{\prime}=\rho^{\prime}$, and so, all together, we get $S \models \pi=\rho$. This shows $\llbracket \Gamma \rrbracket \subseteq \mathrm{V}$.

\section{BREAKING FACTORIZATIONS ASSUMING BIDETERMINISTIC CLOSURE}

In this section we see how closure under bidetermistic product allows us to decompose the pseudoidentities found in Corollary 6.11 into pieces involving only products of regular elements over the organizing pseudovariety.

Proposition 7.1. Consider a semigroup pseudovariety closed under bideterministic product. Let $\pi \in \bar{\Omega}_{A} \mathrm{~S}$. A factorization of $\pi$ is organized with short $\mathrm{V}$-breaks if and only if it is organized with long $\mathrm{V}$-breaks.

Proof. Recall that the "if" part of the theorem is immediate (Remark 6.7).

Conversely, consider a factorization

$$
\pi=u_{0} \cdot \pi_{1} \cdot u_{1} \cdot \pi_{2} \cdots \pi_{n-1} \cdot u_{n-1} \cdot \pi_{n} \cdot u_{n},
$$

that is organized with short $\mathrm{V}$-breaks. We prove by induction on $n \geq 0$ that it is organized with long $\mathrm{V}$-breaks. The base case $n=0$ holds trivially. Suppose that $n>0$ and that the theorem holds for smaller values of $n$.

We show that Conditions (LB.1) and (LB.4) in Definition 6.6 hold for (7.1). Since the factorization $u_{0} \cdot \pi_{1} \cdot u_{1} \cdot \pi_{2} \cdots \pi_{n-1} \cdot u_{n-1}$ is clearly organized with short V-breaks, it is, by the induction hypothesis, organized with long V-breaks. Therefore, to establish Conditions (LB.1) and (LB.4) in Definition 6.6 for the factorization (7.1), it only remains to show that it is impossible to have $u_{n} \neq 1$ and

$$
\left[u_{0} \pi_{1} u_{1} \pi_{2} \cdots \pi_{n-1} u_{n-1} \pi_{n} \mathrm{i}_{1}\left(u_{n}\right)\right]_{\mathrm{V}} \mathcal{R}\left[u_{0} \pi_{1} u_{1} \pi_{2} \cdots \pi_{n-1} u_{n-1} \pi_{n}\right]_{\mathrm{V}} .
$$

Suppose, on the contrary, that that is possible. Take $\rho=u_{0} \pi_{1} u_{1} \pi_{2} \cdots \pi_{n-1}$ and $u_{n}=b w$, with $b \in A$ and $w \in A^{*}$. Then, by Lemma 4.4, for every sufficiently large positive integer $k$, the sets

$$
\left[B\left([\rho]_{\vee}, \frac{1}{k}\right) \cap A^{*}\right] \cdot u_{n-1} \quad \text { and } \quad\left[B\left(\left[\pi_{n}\right]_{\vee}, \frac{1}{k}\right) \cap A^{*}\right] \cdot b
$$

are prefix codes. Therefore, for every sufficiently large $k$, their product

$$
P_{k}=\left[B\left([\rho]_{\mathrm{v}}, \frac{1}{k}\right) \cap A^{*}\right] \cdot u_{n-1} \cdot\left[B\left(\left[\pi_{n}\right]_{\mathrm{v}}, \frac{1}{k}\right) \cap A^{*}\right] \cdot b
$$

is a prefix code. Following the notation of Proposition 4.7, note that

$$
P_{k}=\left[L_{k}\left([\rho]_{\vee}, u_{n-1},\left[\pi_{n}\right]_{\vee}\right) \cap A^{+}\right] \cdot b .
$$

On the other hand, since $\left([\rho]_{\vee}, u_{n-1},\left[\pi_{n}\right]_{\mathrm{V}}\right)$ is a +-good factorization, the language $L_{k}\left([\rho]_{\mathrm{V}}, u_{n-1},\left[\pi_{n}\right]_{\mathrm{V}}\right) \cap A^{+}$is $\mathrm{V}$-recognizable for every sufficiently large $k$ (cf. Proposition 4.7). Therefore, again applying the hypothesis that $\mathrm{V}$ is closed under bideterministic product, we conclude that $P_{k}$ is a 
V-recognizable language, and so the closure ${\overline{P_{k}}}^{\mathrm{V}}$ of $P_{k}$ in $\bar{\Omega}_{A} \mathrm{~V}$ is a clopen subset of $\bar{\Omega}_{A} \mathrm{~V}$. Since

$$
{\overline{P_{k}}}^{\vee}=B\left([\rho]_{\mathrm{V}}, \frac{1}{k}\right) \cdot u_{n-1} \cdot B\left(\left[\pi_{n}\right]_{\mathrm{\vee}}, \frac{1}{k}\right) \cdot b,
$$

we have $\left[\rho \cdot u_{n-1} \cdot \pi_{n} \cdot b\right]_{\vee} \in{\overline{P_{k}}}^{\vee}$. As we are assuming that $(\overline{7.2})$, holds, there is $x \in \bar{\Omega}_{A} \mathrm{~S}$ such that $\left[\rho \cdot u_{n-1} \cdot \pi_{n}\right]_{\mathrm{V}}=\left[\rho \cdot u_{n-1} \cdot \pi_{n} \cdot b x\right]_{\mathrm{V}}$ and thus

$$
\left[\rho \cdot u_{n-1} \cdot \pi_{n} \cdot b\right]_{\mathrm{V}}=\left[\rho \cdot u_{n-1} \cdot \pi_{n} \cdot b x b\right]_{\mathrm{V}} .
$$

Let $\left(\rho_{m}\right)_{m},\left(\pi_{n, m}\right)_{m}$ and $\left(x_{m}\right)_{m}$ be sequences of elements of $A^{+}$respectively converging in $\bar{\Omega}_{A} S$ to $\rho, \pi_{n}$ and $x$, and let $w_{m}=\rho_{m} \cdot u_{n-1} \cdot \pi_{n, m} \cdot b x_{m} b$. Note that, for every sufficiently large $m$, one has $\rho_{m} \in B\left([\rho]_{\mathrm{V}}, \frac{1}{k}\right)$ and also $\pi_{n, m} \in B\left(\left[\pi_{n}\right]_{\mathrm{V}}, \frac{1}{k}\right)$, thus $\rho_{m} \cdot u_{n-1} \cdot \pi_{n, m} \cdot b \in P_{k}$ and $w_{m} \in P_{k} \cdot A^{+}$. On the other hand, by (17.3) , the sequence of words $\left(w_{m}\right)_{m}$ converges in $\bar{\Omega}_{A} \vee$ to $\left[\rho \cdot u_{n-1} \cdot \pi_{n} b\right]_{\mathrm{V}}$. Since the latter has ${\overline{P_{k}}}^{\vee}$ as a neighborhood, we conclude that for sufficiently large $m$ the word $w_{m}$ belongs to the intersection $P_{k} \cap P_{k} A^{+}$. But this contradicts $P_{k}$ being a prefix code. Therefore, in order to avoid this contradiction, one must not have (7.2) whenever $u_{n} \neq 1$.

We established Conditions (LB.1) and (LB.4) in Definition 6.6 for the factorization (7.1). Symmetrically, Conditions [LB.2) and (LB.3) hold for the same factorization. This concludes the inductive step.

We are now ready for showing the central result of this paper.

Theorem 7.2. Let $\mathrm{\vee}$ be a semigroup pseudovariety closed under bideterministic product. Take $\pi, \rho \in \bar{\Omega}_{A} \mathrm{~S}$ such that $\mathrm{V} \models \pi=\rho$. If

$$
\begin{aligned}
& \pi=u_{0} \cdot \pi_{1} \cdot u_{1} \cdot \pi_{2} \cdots \pi_{n-1} \cdot u_{n-1} \cdot \pi_{n} \cdot u_{n}, \\
& \rho=v_{0} \cdot \rho_{1} \cdot v_{1} \cdot \rho_{2} \cdots \rho_{m-1} \cdot v_{m-1} \cdot \rho_{m} \cdot v_{m},
\end{aligned}
$$

are factorizations multiregularly organized in $\mathrm{V}$ and with short $\mathrm{V}$-breaks, then $n=m, u_{i}=v_{i}$ and $\vee \models \pi_{j}=\rho_{j}$ for every $i \in\{0,1, \ldots, n\}$ and $j \in\{1, \ldots, n\}$.

Proof. If $n=0$, then $\pi \in A^{+}$. Since $\mathrm{N} \models \pi=\rho$, we then must have $\pi=\rho$, $m=0$ and $u_{0}=\pi=\rho=v_{0}$.

Suppose that $n, m \geq 1$. We prove the theorem by induction on

$$
s=n+m+\sum_{i=0}^{n}\left|u_{i}\right|+\sum_{j=0}^{m}\left|v_{j}\right| .
$$

Note that $s \geq n+m \geq 2$. If $s=2$, then $n=m=1$ and $u_{i}=v_{j}=1$ for every possible $i, j$. Therefore, $\mathrm{V} \models \pi=\pi_{1}=\rho_{1}=\rho$ and the theorem holds in the base case $s=2$.

Suppose that $s>2$, and suppose that the theorem holds for smaller values of $s$. We consider the (possibly empty) pseudowords

$$
\bar{\pi}=\pi_{2} \cdots \pi_{n-1} \cdot u_{n-1} \cdot \pi_{n} \cdot u_{n} \quad \text { and } \quad \bar{\rho}=\rho_{2} \cdots \rho_{m-1} \cdot v_{m-1} \cdot \rho_{m} \cdot v_{m} .
$$


Note that, according to Proposition 7.1, both factorizations (7.4) and (7.5) are multiregularly organized with long V-breaks. Therefore, if $u_{0}=u^{\prime} a$, with $a \in A$ and $u^{\prime} \in A^{*}$, then $\left(u^{\prime}, a,\left[\pi_{1} u_{1} \bar{\pi}\right] \mathrm{V}\right)$ is a good factorization, and if $v_{0}=v^{\prime} b$, with $b \in A$ and $v^{\prime} \in A^{*}$, then $\left(v^{\prime}, b,\left[\rho_{1} u_{1} \bar{\rho}\right] \mathrm{V}\right)$ is a good factorization. Then, taking into account that both $\pi_{1}$ and $\rho_{1}$ have regular elements of $\bar{\Omega}_{A} \mathrm{~V}$ as prefixes (they are $\mathrm{V}$-multiregulars), applying Lemma 5.11 we conclude that $u_{0}=1$ if and only if $v_{0}=1$.

Suppose that $u_{0} \neq 1$ and $v_{0} \neq 1$. Without loss of generality, assume that $\left|u_{0}\right| \leq\left|v_{0}\right|$. Consider the factorization $u_{0}=u^{\prime} a$ such that $a \in A$ and $u^{\prime} \in A^{*}$, and the factorization $v_{0}=v^{\prime} b v^{\prime \prime}$ such that $b \in A, v^{\prime}, v^{\prime \prime} \in A^{*}$ and $\left|v^{\prime}\right|=\left|u^{\prime}\right|$. Since $u^{\prime}$ and $v^{\prime}$ are finite words, when we use Theorem 4.9 to compare the factorizations $\left(u^{\prime}, a,\left[\pi_{1} u_{1} \bar{\pi}\right]_{\mathrm{V}}\right)$ and $\left(v^{\prime}, b,\left[v^{\prime \prime} \rho_{1} v_{1} \bar{\rho}\right]_{\mathrm{V}}\right)$ of $[\pi]_{\mathrm{V}}=[\rho]_{\mathrm{V}}$, the first of which is a good one, the only possibility is that $u^{\prime}=v^{\prime}, a=b$, and

$$
\mathrm{V} \models \pi_{1} \cdot u_{1} \cdot \pi_{2} \cdots \pi_{n-1} \cdot u_{n-1} \cdot \pi_{n} \cdot u_{n}=v^{\prime \prime} \cdot \rho_{1} \cdot v_{1} \cdot \rho_{2} \cdots \rho_{n-1} \cdot v_{n-1} \cdot \rho_{m} \cdot v_{m}
$$

Since

$$
\begin{aligned}
& \left(n+m+\sum_{i=0}^{n}\left|u_{i}\right|+\sum_{j=0}^{m}\left|v_{j}\right|\right)-\left(n+m+\sum_{i=1}^{n}\left|u_{i}\right|+\left|v^{\prime \prime}\right|+\sum_{j=1}^{m}\left|v_{j}\right|\right)= \\
= & \left|u_{0}\right|+\left|v_{0}\right|-\left|v^{\prime \prime}\right|=\left|u_{0}\right|+\left|v^{\prime}\right|+1>0,
\end{aligned}
$$

we may apply in (7.6) the induction hypothesis, from which we conclude that $n=m, v^{\prime \prime}=1, u_{i}=v_{i}$ and $\mathrm{V} \models \pi_{j}=\rho_{j}$ for every $i \in\{0,1, \ldots, n\}$ and $j \in\{1, \ldots, n\}$. Therefore, we may suppose from hereon that $u_{0}=v_{0}=1$.

Suppose that $u_{1}=1$ also holds (the case $v_{1}=1$ is symmetric). Note that then one has $n=1$. Moreover, $[\pi]_{\vee}=[\rho]_{\vee}$ has no good factorizations (cf. Corollary 5.2), whence it has no +-good factorization (cf. Lemma 4.6). The latter implies $m=1, v_{0}=v_{1}=1$. Hence, the theorem holds in this case.

Finally, we suppose that $u_{1} \neq 1$ and $v_{1} \neq 1$. Consider factorizations $u_{1}=c \bar{u}_{1}$ with $c \in A$ and $\bar{u}_{1} \in A^{*}$, and $v_{1}=d \bar{v}_{1}$ with $d \in A$ and $\bar{v}_{1} \in A^{*}$. Compare the factorizations $\left(\left[\pi_{1}\right]_{\mathrm{V}}, c,\left[\bar{u}_{1} \bar{\pi}\right]_{\mathrm{V}}\right)$ and $\left(\left[\rho_{1}\right]_{\mathrm{V}}, d,\left[\bar{v}_{1} \bar{\rho}\right]_{\mathrm{V}}\right)$. They are good, by Lemma 4.6. By Theorem 4.9, one of the following cases holds:

(1) $\left[\pi_{1}\right]_{\mathrm{V}}=\left[\rho_{1}\right]_{\mathrm{V}}, c=d$ and $\left[\bar{u}_{1} \bar{\pi}\right]_{\mathrm{V}}=\left[\bar{v}_{1} \bar{\rho}\right]_{\mathrm{V}}$;

(2) $\left[\pi_{1}\right]_{\mathrm{V}}=\left[\rho_{1} d t\right]_{\mathrm{V}}$ and $\left[t c \bar{u}_{1} \bar{\pi}\right]_{\mathrm{V}}=\left[\bar{v}_{1} \bar{\rho}\right]_{\mathrm{V}}$, for some $t \in\left(\bar{\Omega}_{A} \mathrm{~S}\right)^{1}$;

(3) $\left[\pi_{1} c t\right]_{\vee}=\left[\rho_{1}\right]_{\vee}$ and $\left[\bar{u}_{1} \bar{\pi}\right]_{\mathrm{V}}=\left[t d \bar{v}_{1} \bar{\rho}\right]_{\mathrm{V}}$, for some $t \in\left(\bar{\Omega}_{A} \mathrm{~S}\right)^{1}$.

Suppose Case (2) holds. Since $\left(\left[\rho_{1}\right]_{\mathrm{V}}, d,\left[t c \bar{u}_{1} \bar{\pi}\right]_{\mathrm{V}}\right)=\left(\left[\rho_{1}\right]_{\mathrm{V}}, d,\left[\bar{v}_{1} \bar{\rho}\right]_{\mathrm{V}}\right)$ is a good factorization of $[\rho]_{\mathrm{V}}$, it follows from Lemma 4.2 that $\left(\left[\rho_{1}\right], d,[t]_{\mathrm{V}}\right)$ is a good factorization of $\left[\rho_{1} d t\right]_{\vee}=\left[\pi_{1}\right]_{\vee}$. But this is impossible in view of Corollary [5.2, because $\left[\pi_{1}\right]_{\vee}$ is a product of regular elements. Therefore, Case (21) does not hold. By symmetry, we conclude that Case (31) is also impossible, and therefore only Case (11) holds. We may then apply the induction hypothesis to

$\mathrm{V} \models \bar{u}_{1} \cdot \pi_{2} \cdot u_{2} \cdot \pi_{3} \cdots \pi_{n-1} \cdot u_{n-1} \cdot \pi_{n} \cdot u_{n}=\bar{v}_{1} \cdot \rho_{2} \cdot v_{2} \cdot \rho_{3} \cdots \rho_{m-1} \cdot v_{m-1} \cdot \rho_{m} \cdot v_{m}$ 
to obtain $\bar{u}_{1}=\bar{v}_{1}$ (and so $u_{1}=v_{1}$ ), $n=m, u_{i}=v_{i}$ and $\mathrm{V} \models \pi_{j}=\rho_{j}$ for every $i \in\{0,1, \ldots, n\}$ and $j \in\{1, \ldots, n\}$.

This exhausts all possibles cases to consider in the inductive step.

Corollary 7.3. Let $\mathrm{V}$ be a pseudovariety of semigroups closed under bideterministic product. Suppose that $\mathrm{W}$ is an organizing pseudovariety containing $\mathrm{V}$. Then $\mathrm{V}$ is multiregularly based in $\mathrm{W}$.

Proof. By Corollary 6.11, V has a basis $\Sigma$ of pseudoidentities multiregularly organized in W (whence in $\mathrm{V}$ ) and with short V-breaks. Let $(\pi=\rho) \in \Sigma$. Suppose that the next factorizations are multiregularly organized in $W$ and have short V-breaks:

$\pi=u_{0} \cdot \pi_{1} \cdot u_{1} \cdot \pi_{2} \cdots u_{n-1} \cdot \pi_{n} \cdot u_{n} \quad$ and $\quad \rho=v_{0} \cdot \rho_{1} \cdot v_{1} \cdot \rho_{2} \cdots v_{m-1} \cdot \rho_{m} \cdot v_{m}$.

By Theorem 7.2, we know that $n=m, u_{i}=v_{i}$ and $\mathrm{V} \models \pi_{j}=\rho_{j}$ for every $i \in\{0,1, \ldots, n\}$ and $j \in\{1, \ldots, n\}$. The integer $n$ depends on $(\pi=\rho)$, and for that reason we denote it by $n_{(\pi=\rho)}$. For each $(\pi=\rho) \in \Sigma$, let $\Gamma_{(\pi=\rho)}=\left\{\left(\pi_{j}=\rho_{j}\right) \mid 1 \leq j \leq n_{(\pi=\rho)}\right\}$. Consider the set of pseudoidentities $\Gamma=\bigcup_{(\pi=\rho) \in \Sigma} \Gamma_{(\pi=\rho)}$. To conclude the proof, it suffices to show that $\mathrm{V}=\llbracket \Gamma \rrbracket$. We already saw that $\mathrm{V} \models \Gamma$. Conversely, suppose that $S$ is a semigroup such that $S \models \Gamma$. Fix a pseudoidentity $(\pi=\rho) \in \Sigma$. For each $j \in\left\{1, \ldots, n_{(\pi=\rho)}\right\}$, we have $S \models \pi_{j}=\rho_{j}$. This clearly implies $S \models \pi=\rho$, in view of the factorizations of $\pi$ and $\rho$ with which we are working with. Hence, we have $S \models \Sigma$, that is, $S \in \mathrm{V}$. This concludes the proof that $\mathrm{V}=\llbracket \Gamma \rrbracket$.

We next highlight the case where the organizing pseudovariety is DS.

Theorem 7.4. Suppose that $\mathrm{V}$ is a semigroup pseudovariety in the interval [SI,DS]. The following conditions are equivalent:

(1) $\mathrm{V}$ is closed under bideterministic product;

(2) $\mathrm{V}$ is multiregularly based in DS;

(3) $\mathrm{V}$ is multiregularly based.

Proof. (1) $\Rightarrow(2)$ : This is a direct application of Corollary 7.3 , in view of the fact that DS is an organizing pseudovariety.

$(2) \Rightarrow(3)$ : If $\pi=\pi_{1} \cdots \pi_{n}$ is a DS-multiregular pseudoword, then the product $\pi^{\prime}=\pi_{1}^{\omega+1} \cdots \pi_{n}^{\omega+1}$ is a multiregular pseudoword for which we have DS $\models \pi=\pi^{\prime}$. Therefore, if $\Sigma$ is a basis for $\bigvee$ formed by pseudoidentities between DS-multiregular pseudowords, then

$$
\Sigma^{\prime}=\left\{\left(\pi^{\prime}=\rho^{\prime}\right) \mid(\pi=\rho) \in \Sigma\right\} \cup\left\{\left((x y)^{\omega}(y x)^{\omega}(x y)^{\omega}\right)^{\omega}=(x y)^{\omega}\right\}
$$

is a basis for $\mathrm{V}$, comprised solely by pseudoidentities between products of S-regular pseudowords.

$(\underline{3}) \Rightarrow(1)$ : It follows from Proposition 5.8 . 


\section{FACtORizations in THE GLOBAL}

8.1. Semigroupoids. For the reader to situate himself better, we give some notation and recall some facts on semigroupoids. We refer to [24, 12, 34].

A semigroupoid $S$ is a graph $V(S) \cup E(S)$ endowed with two operations $\alpha, \omega: E(S) \rightarrow V(S)$ which give respectively the beginning and end vertices of each edge, and a partial associative multiplication on $E(S)$ given by: for $s, t \in E$, st is defined if and only if $\omega(s)=\alpha(t)$ and, then, $\alpha(s t)=\alpha(s)$ and $\omega(s t)=\omega(t)$. For a graph $\Gamma$, the free semigroupoid $\Gamma^{+}$on $\Gamma$ has as vertex-set $V(\Gamma)$ and as edges the non-empty paths on $\Gamma$.

Every semigroup $S$ may be viewed as a semigroupoid by taking the set of edges $S$ with both ends at an added vertex. Conversely, for a semigroupoid $S$ and a vertex $v$ of $S$, the set $S(v)$ of all loops at vertex $v$ constitutes an semigroup called the local semigroup of $S$ at $v$.

A pseudovariety of semigroupoids is a class of finite semigroupoids closed under taking divisors of semigroupoids, and finitary direct products. The pseudovariety of all finite semigroupoids is denoted by Sd.

From hereon, we assume that all semigroupoids have a finite number of vertices. A compact semigroupoid $S$ is a semigroupoid endowed with a compact topology on $E(S)$ and the discrete topology in the finite set $V(S)$, with respect to which the partial operations $\alpha, \omega$, and edge multiplication are continuous (see 6] for delicate questions related with infinite-vertex semigroupoids). Finite semigroupoids equipped with the discrete topology become compact semigroupoids. Let $\mathrm{V}$ be a pseudovariety of semigroupoids. A compact semigroupoid is pro- $\mathrm{V}$ if it is compact and every pair of distinct coterminal edges $u$ and $v$ can be separated by a continuous semigroupoid homomorphism into a semigroupoid of $\mathrm{V}$. For a finite graph $\Gamma$, a compact semigroupoid $S$ is $\Gamma$-generated if there is a graph homomorphism $\varphi: \Gamma \rightarrow S$ such that the subgraph of $S$ generated by $\varphi(\Gamma)$ is dense. We denote by $\bar{\Omega}_{\Gamma} \mathrm{V}$ the free pro- $\vee \Gamma$-generated semigroupoid. The semigroupoid $\bar{\Omega}_{\Gamma} \mathrm{V}$ has the usual universal property.

For each finite graph $A$, the free semigroupoid $A^{+}$generated by $A$ is dense in $\bar{\Omega}_{A} \mathrm{Sd}$. The edges of $A^{+}$are the nonempty paths on $A$, whence the name pseudopath for the edges of $\bar{\Omega}_{A} \mathrm{Sd}$. A generalization of Reiterman's Theorem states that the pseudovarieties of semigroupoids are the classes of finite semigroupoids defined by pseudoidentities, that is formal identities between pseudopaths of finitely generated free pro-Sd semigroupoids ([24, 12]).

We are mostly interested in two kinds of semigroupoid pseudovarieties induced by a semigroup pseudovariety $\mathrm{V}$ : the pseudovariety $g \mathrm{~V}$ of semigroupoids generated by $\mathrm{V}$ (the global of $\mathrm{V}$ ), and the pseudovariety $\ell \mathrm{V}$ of semigroupoids whose local semigroups belong to $\mathrm{V}$. One has $g \mathrm{~V} \subseteq \ell \mathrm{V}$, and if the equality holds, then the pseudovariety $\mathrm{V}$ is said to be local.

Let $A$ be a finite graph. In general a pseudoidentity between edges $\pi$ and $\rho$ of $\bar{\Omega}_{A} \mathrm{Sd}$ is denoted $(A ; \pi=\rho)$, because if $A$ is a subgraph of $B$, then a finite semigroupoid satisfying $(B ; \pi=\rho)$ may not satisfy $(A ; \pi=\rho)$, see [34, 
pages 100 and 101]. However, if $\mathrm{V}$ is a semigroup pseudovariety, then we can write a pseudoidentity $(A ; \pi=\rho)$ satisfied by $\bar{\Omega}_{A} g \vee$ simply by $(\pi=\rho)$, because in that case there is no dependence on $A$ [34, Theorem 2.5.15].

8.2. Semigroupoid versions of previous definitions and results. Several basic concepts for semigroups carry on to semigroupoids without substantial modifications. For example, in a semigroupoid $S$ one may consider the Green relations between edges, an edge $s$ is regular if $s=s x s$ for some edge $x$ of $S$, etc. Definitions 6.6 and 6.8 also carry on to (pseudovarieties of) semigroupoids with no real modifications: just replace pseudowords by pseudopaths, and words by paths. For example, an edge $\pi$ of $\bar{\Omega}_{A} \mathrm{Sd}$ is V-regular if its canonical projection $[\pi]_{\vee}$ in $\bar{\Omega}_{A} \vee$ is regular, where $\mathrm{V}$ is a semigroupoid pseudovariety. Next are the semigroupoid versions of Propositions 6.10 and Corollary 6.11, for which entirely analogous proofs hold.

Proposition 8.1. Let $\mathrm{V}$ be a pseudovariety of semigroupoids and let $A$ a finite graph. Suppose that $\pi$ is an edge of $\bar{\Omega}_{A} \mathrm{Sd}$ with a factorization multiregularly organized in $\mathrm{W}$, where $\mathrm{W}$ is a semigroupoid pseudovariety. Then there is an edge $\pi^{\prime}$ in $\bar{\Omega}_{A} \mathrm{Sd}$ and a set $\Upsilon_{\pi}$ of pseudoidentities over $A$ satisfying the following conditions:

(1) $\vee \subseteq \llbracket \Upsilon_{\pi} \rrbracket \subseteq \llbracket\left(A ; \pi=\pi^{\prime}\right) \rrbracket$;

(2) $\pi^{\prime}$ has a factorization multiregularly organized in $\mathrm{W}$ and with short $\mathrm{V}$-breaks;

(3) if $(A ; \varpi=\varrho)$ belongs to $\Upsilon_{\pi}$, then $\varpi$ and $\varrho$ are $\mathrm{W}$-multiregulars.

Corollary 8.2. Let $\mathrm{V}$ and $\mathrm{W}$ be pseudovarieties of semigroupoids, with $\mathrm{W}$ being an organizing pseudovariety. Then $\mathrm{V}$ has a basis of pseudoidentities with factorizations multiregularly organized in $\mathrm{W}$ and with short $\mathrm{V}$-breaks.

8.3. The interval [SI, DS $\cap \mathrm{RS}]$. We denote by RS the class of finite semigroups $S$ whose set of regular elements is a subsemigroup of $S$. Although $\mathrm{RS}$ is not a pseudovariety, the class DS $\cap \mathrm{RS}$ is a semigroup pseudovariety, with

$$
\mathrm{RS} \cap \mathrm{DS}=\mathrm{DS} \cap \llbracket x^{\omega+1} y^{\omega+1}=\left(x^{\omega+1} y^{\omega+1}\right)^{\omega+1} \rrbracket,
$$

as the regular elements of a semigroup of DS are its group elements. Note also that (8.1) yields the following corollary of Proposition 5.8

Corollary 8.3. The pseudovariety DS $\cap \mathrm{RS}$ is closed under bideterministic product.

Denote by $\operatorname{Reg}(S)$ the subgraph, of the semigroupoid $S$, formed by the regular edges of $S$. Let RSd be the class of finite semigroupoids $S$ such that $\operatorname{Reg}(S)$ is a subsemigroupoid of $S$. Here $\ell$ RS is the class of finite semigroupoids whose local semigroups belong to RS.

Proposition 8.4. The equality $\ell \mathrm{RS}=\mathrm{RSd}$ holds. 
Proof. Clearly, for every semigroupoid $S$, and every vertex $v$ of $S$, an element of the local semigroup $S_{v}$ of $S$ at $v$ is regular in $S_{v}$ if and only if it is regular in $S$. Therefore, the inclusion RSd $\subseteq \ell \mathrm{RS}$ is immediate.

Conversely, let $S \in \ell \mathrm{RS}$, and let $s, t$ be consecutive regular edges of $S$. Take edges $x$ and $y$ such that $s=s x s$ and $t=t y t$. Note that $x s$ and $t y$ are idempotents rooted at the vertex $v=\omega s$. Since $x s$ and $t y$ are regular elements of the local semigroup $S_{v}$ at $v$, we know that $x s \cdot t y=x s \cdot t y \cdot z \cdot x s \cdot t y$ for some loop $z$ belonging to $S_{v}$. We then have

$s t=s \cdot x s \cdot t y \cdot t=s \cdot x s \cdot t y \cdot z \cdot x s \cdot t y \cdot t=s x s \cdot t \cdot y z x \cdot s \cdot t y t=s t \cdot y z x \cdot s t$, thus showing that the edge $s t$ is regular in $S$.

Corollary 8.5. For any finite graph A, every product of regular edges of $\bar{\Omega}_{A} \ell(\mathrm{DS} \cap \mathrm{RS})$ is a regular edge of $\bar{\Omega}_{A} \ell(\mathrm{DS} \cap \mathrm{RS})$.

8.4. Honest pseudovarieties. Recall that a semigroupoid homomorphism is faithful if it maps distinct coterminal edges to distinct coterminal edges.

Proposition 8.6 ([2]). If $\mathrm{V}$ is a pseudovariety of semigroups, then the unique continuous semigroupoid homomorphism from $\bar{\Omega}_{A} g \mathrm{~V}$ onto $\bar{\Omega}_{E(A)} \mathrm{V}$ that maps $[a]_{g \vee}$ to $[a]_{\vee}$, for every $a \in E(A)$, is a faithful homomorphism.

The next proposition brings nothing new, but we did not find a direct reference for it. The content $c(\pi)$ of a pseudopath $\pi$ of $\bar{\Omega}_{A} \mathrm{Sd}$ is the subgraph $X \cup \alpha(X) \cup \omega(X)$ of $A$ where $X \subseteq E(A)$ satisfies $c\left([\pi]_{\mathrm{S}}\right)=\left\{[a]_{\mathrm{S}} \mid a \in X\right\}$. For pseudopaths $\pi, \rho$, we write $c(\rho) \subseteq c(\pi)$ when $c(\rho)$ is a subgraph of $c(\pi)$.

Proposition 8.7. Let $\mathrm{V}$ be a pseudovariety of semigroupoids in the interval $[g \mathrm{SI}, g \mathrm{DS}]$. Let $A$ be a finite graph. Suppose that the edge $\pi$ of $\bar{\Omega}_{A} \mathrm{Sd}$ is regular in $\mathrm{V}$, and let $\rho$ be an edge of $\bar{\Omega}_{A} \mathrm{Sd}$ such that $\omega(\pi)=\alpha(\rho)$. Then we have $\mathrm{V} \models \pi \rho \mathcal{R} \pi$ if and only if $c(\rho) \subseteq c(\pi)$.

Proof. If $\mathrm{V} \models \pi \rho \mathcal{R} \pi$, then $\mathrm{SI} \models \pi \rho \mathcal{R} \pi$ holds because $\mathrm{V}$ contains $g \mathrm{SI}$, whence $c(\rho) \subseteq c(\pi)$. Conversely, suppose that $c(\rho) \subseteq c(\pi)$. As $\pi$ is $\mathrm{V}$-regular, there is $z \in \bar{\Omega}_{A} \mathrm{Sd}$ such that $\mathrm{V} \models \pi=\pi z \pi$ (which implies that $[z \pi]_{\mathrm{V}}$ is idempotent), and the graph $c(\pi)$ is strongly connected. Therefore, and since $c(\rho) \subseteq c(\pi)$, there is a path $v$ in $c(\pi)$ such that $\alpha(v)=\omega(\rho)$ and $\omega(v)=\alpha(z)$. We may then consider the idempotent loop $s=\left((z \pi)^{\omega} \rho v(z \pi)^{\omega}\right)^{\omega}$ of $\bar{\Omega}_{A} \mathrm{Sd}$. Because $c(\rho) \subseteq c(\pi)$, we also have $c(\pi)=c(s)=c(z \pi)$ and DS $\models s=(z \pi)^{\omega}$. Applying Proposition [8.6. we get $g \mathrm{DS} \models s=(z \pi)^{\omega}$, thus $\mathrm{V} \models s=z \pi$. Therefore, $\mathrm{V} \models \pi=\pi s=\pi \rho v z \pi(\rho v z \pi)^{\omega-1}$, establishing $\mathrm{V} \models \pi \mathcal{R} \pi \rho$.

Definition 8.8. A pseudovariety of semigroups $\mathrm{V}$ is honest if, for every finite graph $A, a \in A$ and $\pi \in \bar{\Omega}_{A} \mathrm{Sd}$ such that $\pi$ is a product of regular elements of $\bar{\Omega}_{A} g \mathrm{\vee}$, the following conditions hold:

(1) when $\omega \pi=\alpha a$, one has $\bigvee \models \pi a \mathcal{R} \pi \Longrightarrow g \bigvee \models \pi a \mathcal{R} \pi$;

(2) when $\omega a=\alpha \pi$, one has $\bigvee \models a \pi \mathcal{L} \pi \Longrightarrow g \bigvee \models a \pi \mathcal{L} \pi$.

Proposition 8.9. The semigroup pseudovarieties in the intervals [J,DS] and $[\mathrm{SI}, \mathrm{DS} \cap \mathrm{RS}]$ are honest. 
Proof. Suppose that $\mathrm{V} \in[\mathrm{J}, \mathrm{DS}]$. Let $\pi$ and $a$ be as in Definition 8.8, with $\omega \pi=\alpha a$ (the case $\alpha \pi=\omega a$ is symmetric). By Lemma 6.3, the projection of $\pi$ in $\bar{\Omega}_{E(A)} \mathrm{S}$, still denoted $\pi$, is a J-reduced multiregular $\pi_{1} \cdots \pi_{n}$ in $\bar{\Omega}_{E(A)} \mathrm{S}$. Applying Lemma 6.5. from $\mathrm{V} \models \pi \mathcal{R} \pi a$ we get $a \in c\left(\pi_{n}\right)$. It follows from Proposition 8.7 that $g \bigvee \models \pi_{n} a \mathcal{R} \pi_{n}$, whence $g \mathrm{~V} \models \pi a \mathcal{R} \pi$.

Finally, if $\mathrm{V} \in[\mathrm{SI}, \mathrm{DS} \cap \mathrm{RS}]$, then a product of regular elements of $\bar{\Omega}_{A} g \mathrm{~V}$ is a regular element of $\bar{\Omega}_{A} g \mathrm{~V}$, by Proposition 8.4. Therefore, $\mathrm{V} \models \pi a \mathcal{R} \pi$ implies $g \bigvee \models \pi a \mathcal{R} \pi$ by Proposition 8.7 also in this case.

We leave open the problem of identifying all the honest pseudovarieties.

Proposition 8.10. Let $\mathrm{V}$ be a honest pseudovariety of semigroups that is closed under bideterministic product. Suppose W is a semigroup pseudovariety containing $\mathrm{V}$ and such that $\mathrm{g} \mathrm{W}$ is an organizing pseudovariety. Then $g \mathrm{~V}$ is multiregularly based in $\mathrm{g} \mathrm{W}$.

Proof. By Corollary 8.2, the global $g \vee$ has a basis $\Sigma$ formed by edge pseudoidentities $(\pi=\rho)$ with pseudopath factorizations

$\pi=u_{0} \cdot \pi_{1} \cdot u_{1} \cdot \pi_{2} \cdots u_{n-1} \cdot \pi_{n} \cdot u_{n} \quad$ and $\quad \rho=v_{0} \cdot \rho_{1} \cdot v_{1} \cdot \rho_{2} \cdots v_{m-1} \cdot \rho_{m} \cdot v_{m}$ that are both organized in $g \mathrm{~W}$ and with short $g \mathrm{~V}$-breaks. Projecting in $\bar{\Omega}_{E(A)} \mathrm{V}$ and seeing these factorizations as pseudowords factorizations, we see that they are multiregularly organized in $\mathrm{W}$ and with short $\mathrm{V}$-breaks, the latter property holding because $\mathrm{V}$ is honest. Combining with $\mathrm{V} \models \pi=\rho$ and Theorem [7.2, we get that $n=m, u_{i}=v_{i}$ and $\mathrm{V} \models \pi_{j}=\rho_{j}$ for every $i \in\{0,1, \ldots, n\}$ and $j \in\{1, \ldots, n\}$. In particular, we have the equalities $\alpha\left(\pi_{j}\right)=\omega\left(u_{j-1}\right)=\omega\left(v_{j-1}\right)=\alpha\left(\rho_{j}\right)$ and $\omega\left(\pi_{j}\right)=\alpha\left(u_{j}\right)=\alpha\left(v_{j}\right)=$ $\omega\left(\rho_{j}\right)$. Therefore, by Proposition [8.6, we have $g \vee \models \pi_{j}=\rho_{j}$, for every $j \in\{1, \ldots, n\}$.

As the integer $n$ depends on $(\pi=\rho)$, we denote it by $n_{(\pi=\rho)}$. For each $(\pi=$ $\rho) \in \Sigma$, consider the set of pseudopath pseudoidentities defined by $\Gamma_{(\pi=\rho)}=$ $\left\{\left(\pi_{j}=\rho_{j}\right) \mid 1 \leq j \leq n_{(\pi=\rho)}\right\}$. Take the union $\Gamma=\bigcup_{(\pi=\rho) \in \Sigma} \Gamma_{(\pi=\rho)}$ Then, in view of the conclusion at which we arrived in the previous paragraph, we have $g \mathrm{~V}=\llbracket \Gamma \rrbracket$, an equality whose detailed justification follows exactly the same argument as in the last lines of the proof of Corollary 7.3 .

Corollary 8.11. Suppose that $\mathrm{V}$ is a pseudovariety closed under bideterministic product and belonging to one of the intervals [SI, DS $\cap \mathrm{RS}]$ or [J, DS]. Then $g \vee$ is multiregularly based.

Proof. The pseudovariety $g$ DS is organizing by [9, Proposition 4.2]. Therefore, by Propositions 8.9 and 8.10, we know that $g \mathrm{~V}$ is multiregularly based in $g$ DS. For each $g$ DS-multiregular pseudopath $\pi=\pi_{1} \cdots \pi_{n}$, take for each $g$ DS-regular pseudopath $\pi_{i}$ a pseudopath $z_{i}$ such that $g \mathrm{DS} \models \pi_{i}=\pi_{i}\left(z_{i} \pi_{i}\right)^{\omega}$, and let $\pi^{\prime}=\pi_{1}\left(z_{1} \pi_{1}\right)^{\omega} \cdots \pi_{n}\left(z_{n} \pi_{n}\right)^{\omega}$. Note that $\pi^{\prime}$ is an Sd-multiregular pseudopath. Therefore, if $\Sigma$ is a basis for $g \vee$ formed by pseudoidentities between $g$ DS-multiregular pseudopaths, then

$$
\Sigma^{\prime}=\left\{\left(\pi^{\prime}=\rho^{\prime}\right) \mid(\pi=\rho) \in \Sigma\right\} \cup\left\{\left((x y)^{\omega}(y x)^{\omega}(x y)^{\omega}\right)^{\omega}=(x y)^{\omega}\right\}
$$


is a basis for $g \mathrm{~V}$, comprised solely by multiregular pseudopaths.

9. Preservation of locality inside the interval [SI, DS $\cap$ RS]

A pseudovariety of semigroups is monoidal if it is of the form $V_{S}$, for some pseudovariety of monoids $\mathrm{V}$.

Theorem 9.1 ([19]). Let $\vee$ be a monoidal pseudovariety of semigroups. If $\mathrm{V}$ is local then the pseudovarieties of semigroups $\mathrm{K}(\mathrm{m} \mathrm{V}, \mathrm{D} \rightarrow \mathrm{V}$ and $\mathcal{L} \mathrm{I}(\mathrm{m} \mathrm{V}$ are also local monoidal pseudovarieties of semigroups.

In this section we show that an analog of Theorem 9.1 is valid for the operator $\mathrm{V} \mapsto \overline{\mathrm{V}}$ when restricted to the interval [SI, DS $\cap \mathrm{RS}$ ]. It already follows from Corollary 3.13 that $\bar{V}$ is monoidal when $V$ is monoidal. To proceed, we need the following lemma.

Lemma 9.2. Let $\mathrm{V}$ be a pseudovariety of semigroups such that $\mathrm{K}(\mathrm{m} \mathrm{V}$ and $\mathrm{D} \times \mathrm{V}$ are local pseudovarieties. If the pseudopaths $\pi$ and $\rho$ are loops such that $g \mathrm{~V} \models \pi=\rho=\pi^{2}$, then we have $\ell(\mathrm{N}: \mathrm{m} \mathrm{V}) \models \pi^{\omega}=\pi^{\omega} \rho=\rho \pi^{\omega}=\rho^{\omega}$.

Proof. By the easy part of the Pin-Weil basis theorem for Mal'cev products [32], and by Proposition [8.6, we have $g(\mathrm{~K} m \mathrm{~V}) \models \pi^{\omega} \rho=\pi^{\omega}$ and $g(\mathrm{D} m \mathrm{~V}) \models \rho \pi^{\omega}=\pi^{\omega}$. Since the intersection $\mathrm{K}(\mathrm{m} \mathrm{V} \cap \mathrm{D} ; \mathrm{V}$ contains (it is actually equal to) the pseudovariety $\mathrm{N} m \mathrm{~V}$, and since $\mathrm{K}(m \mathrm{~V}$ and $\mathrm{D}(\mathrm{m} \mathrm{V}$ are local by hypothesis, it follows in particular that $\ell(\mathrm{N} m \mathrm{~V}) \models \pi^{\omega} \rho=\pi^{\omega}=\rho \pi^{\omega}$. The latter implies

$$
\ell(\mathrm{N} \cdot \mathrm{V} \mathrm{V}) \models \pi^{\omega} \rho^{\omega}=\pi^{\omega}=\rho^{\omega} \pi^{\omega} .
$$

On the other hand, the hypothesis $g \bigvee \models \pi=\rho=\pi^{2}$ is clearly equivalent to $g \vee \models \pi=\rho=\rho^{2}$, and so we can interchange the roles of $\pi$ and $\rho$ in (9.1), which altogether implies $\ell(\mathrm{N}: \mathrm{V} \mathrm{V}) \models \pi^{\omega}=\rho^{\omega} \pi^{\omega}=\rho^{\omega}$.

The next proposition is the key to obtain the result we search for.

Proposition 9.3. Let $\mathrm{V}$ be a pseudovariety of semigroups. If $\mathrm{K}(\mathrm{m} \mathrm{V}$ and $\mathrm{D}(\mathrm{m} \mathrm{V}$ are local, and if $g \mathrm{~V}$ has a basis of pseudoidentities between pseudopaths that are regular in $\ell \mathrm{V}$, then $\mathrm{V}$ is local.

Proof. Let $(\pi=\rho)$ be a pseudoidentity satisfied by $g \vee$ such that $\pi$ and $\rho$ are regular in $\ell \mathrm{V}$. The proof amounts to show that $\ell \mathrm{V} \models \pi=\rho$ holds.

We may take a pseudopath $x$ such that

$$
\ell \mathrm{V} \models \pi=\pi x \pi \quad \text { and } \quad \ell \mathrm{V} \models x=x \pi x,
$$

and a pseudopath $y$ such that

$$
\ell \mathrm{V} \models \rho=\rho y \rho \quad \text { and } \quad \ell \mathrm{V} \models y=y \rho y .
$$

As $g \vee \models \pi=\rho$, we have $g \bigvee \models \pi x=\rho x$. Applying Lemma 9.2 to the latter (note that $\ell \mathrm{V} \subseteq \ell(\mathrm{N} m \mathrm{~V})$ ) and observing that $\pi x$ is idempotent in $\ell \mathrm{V}$, we obtain

$$
\ell \mathrm{V} \models \pi x=\pi x \cdot \rho x=\rho x \cdot \pi x
$$


In the pseudovariety $\ell \mathrm{V}$, the pseudopath $\rho y$ is an idempotent which is a prefix of $\rho$, and so, in view of (9.3), a prefix of $\pi$ also. Therefore, we have

$$
\ell \mathrm{V} \models \pi=\rho y \pi \text {. }
$$

Dually, we also have

$$
\ell \mathrm{V} \models \pi=\pi y \rho .
$$

It follows from these facts that the pseudopath $x^{\prime}=y \rho \cdot x \cdot \rho y$ satisfies in $\ell \mathrm{V}$ the pseudoidentities $\pi=\pi x^{\prime} \pi$ and $x^{\prime}=x^{\prime} \pi x^{\prime}$. Moreover, in $\ell \mathrm{V}$ we also have $x^{\prime}=y \rho \cdot x^{\prime} \cdot \rho y$. Therefore, if necessary replacing $x$ by $x^{\prime}$, we may suppose, as we do from hereon, that $x$ not only satisfies (9.2) and (9.3), but also $\ell \mathrm{V} \models x=y \rho \cdot x \cdot \rho y$. In particular, $g \mathrm{~V} \models x=y \rho x \rho y=y \pi x \pi y=$ $y \pi y=y \rho y=y$ holds, whence $g \vee \models x \pi=y \rho$. Then, as both $x \pi$ and $y \rho$ are idempotents in $\ell \mathrm{V}$, applying Lemma 9.2 we obtain $\ell \mathrm{V} \models x \pi=y \rho$. It follows that $\ell \mathrm{V} \models \rho=\rho y \rho=\rho x \pi=\rho x(\pi x \pi)=(\rho x \pi x) \pi$. Replacing in the last term of this chain of pseudoidentities $\rho x \pi x$ by $\pi x$ by means of (9.3), we get $\ell \mathrm{V} \models \rho=\pi x \pi=\pi$, concluding the proof.

We are now ready to deduce the main result of this section.

Theorem 9.4. Let $\mathrm{V}$ be a monoidal pseudovariety of semigroups in the interval [SI, DS $\cap \mathrm{RS}]$. If $\mathrm{V}$ is local, then $\overline{\mathrm{V}}$ is local.

Proof. By Theorem 9.1, both K $m \mathrm{~V}$ and D $m \mathrm{~V}$ are local. By Theorem 2.4, $\mathrm{K}(\mathrm{m} \mathrm{V}$ and $\mathrm{D} m \mathrm{~V}$ are closed under bideterministic product, so

$$
\overline{\mathrm{V}} \subseteq \overline{\mathrm{K} m \mathrm{~V}}=\mathrm{K} m \mathrm{~V} \text { and } \mathrm{K} m \overline{\mathrm{V}} \subseteq \mathrm{K} m(\mathrm{~K} m \mathrm{~V})=\mathrm{K} m \mathrm{~V}
$$

Then we have the equality $\mathrm{K} m \mathrm{~V}=\mathrm{K} m \overline{\mathrm{V}}$ and, by duallity, $\mathrm{D} m \mathrm{~V}=\mathrm{D} m \overline{\mathrm{V}}$.

By Corollary 8.11, $g \overline{\mathrm{V}}$ has a basis of pseudoidentities between multiregular pseudopaths. On the other, note that $\bar{V} \subseteq \mathrm{DS} \cap \mathrm{RS}$, as $\mathrm{DS} \cap \mathrm{RS}$ is closed under bideterministic product. In view of Proposition 8.4, it follows that $g \overline{\mathrm{V}}$ has a basis of pseudoidentities between pseudopaths that are regular in $\ell(D S \cap R S)$, and thus in $\ell \overline{\mathrm{V}}$.

Altogether, the result then follows directly from Proposition 9.3.

Let $\mathrm{G}$ be the pseudovariety of finite groups and let $A b$ be the pseudovariety of finite Abelian groups. It is proved in [26] that DG is local, but not DAb. In contrast, we have the following.

Corollary 9.5. For every pseudovariety $\mathrm{H}$ of finite groups, the pseudovariety $\mathrm{DH} \cap \mathrm{ECom}$ is local.

Proof. It is shown in 25] that SI $\vee \mathrm{H}$ is local. Applying Theorem 9.4, we deduce that $\mathrm{DH} \cap \mathrm{ECom}$ is local (cf. Example 3.8).

Taking into account that every pseudovariety of bands is local [25], or that $\mathrm{CR} \cap \mathrm{H}$ is local for every pseudovariety $\mathrm{H}$ of groups 23, one finds in Example 3.9 other local pseudovarieties resulting from Theorem 9.4. 


\section{INTERPLAY WITH THE SEMIDIRECT PRODUCT WITH D}

Let $\alpha$ be a unary operator on a sublattice of semigroup pseudovarieties containing SI, mapping monoidal pseudovarieties to monoidal pseudovarieties. A monoidal pseudovariety of semigroups $\mathrm{V}$ containing $\mathrm{SI}$ is local if and only if $\mathcal{L} \mathrm{V} \subseteq \mathrm{V} * \mathrm{D}$, if and only if $\mathcal{L} \mathrm{V}=\mathrm{V} * \mathrm{D}$ [36]. Therefore, $\alpha(\mathrm{V})$ is local if $\mathrm{V}$ is local and the following inclusions hold:

$$
\mathcal{L}(\alpha(\mathrm{V})) \subseteq \alpha(\mathcal{L V}) \quad \text { and } \quad \alpha(\mathrm{V} * \mathrm{D}) \subseteq \alpha(\mathrm{V}) * \mathrm{D} .
$$

Theorem 9.1 was proved in [19] using this strategy. Indeed, these inclusions hold for each pseudovariety of semigroups $\mathrm{V}$ containing $\mathrm{SI}$ when $\alpha$ is one of the operators $\mathrm{V} \mapsto \mathrm{K} m \mathrm{~V}$ or $\mathrm{V} \mapsto \mathrm{D} m \mathrm{~V}$. Other operators were considered in [19]. As observed in [19], for the operator $\mathrm{V} \mapsto \mathrm{N} m \mathrm{~V}$ the first inclusion holds when $\mathrm{V} \supseteq \mathrm{SI}$, while the second fails for $\mathrm{V}=\mathrm{SI}$. In this section we verify that the strategy used in [19] does not work for the operator $\mathrm{V} \mapsto \overline{\mathrm{V}}$. While $\overline{\mathrm{V} * \mathrm{D}} \subseteq \overline{\mathrm{V}} * \mathrm{D}$ holds for many subpseudovarieties of DS (cf. Corollary 10.4), including $\mathrm{SI}$, we have $\mathcal{L} \overline{\mathrm{V}} \nsubseteq \overline{\mathcal{L V}}$ when $\mathrm{V}=\mathrm{SI}$. The latter follows from the next proposition, in view of the equality $\mathrm{J} \cap \mathrm{ECom}=\overline{\mathrm{SI}}$ (cf. Example 3.8).

Proposition 10.1. The pseudovariety $\overline{\mathcal{L S I}}$ is strictly contained in $\mathcal{L}(\mathrm{J} \cap \mathrm{ECom})$.

Before we proceed to the proof, recall from [1, Section 10.6] the so called $k$-superposition homomorphism $\Phi_{k}: \bar{\Omega}_{A} \mathrm{~S} \rightarrow\left(\bar{\Omega}_{A^{k+1}} \mathrm{~S}\right)^{1}$, the unique continuous extension of the mapping $A^{+} \rightarrow\left(A^{k+1}\right)^{*}$ that sends words of length at most $k$ into the empty word and reads the consecutive factors of length $k+1$ in every word over $A$ with length at least $k+1$. In the proof of Proposition 10.1 we only need to consider the case $k=1$.

Proof of Proposition 10.1. The inclusion $\overline{\mathcal{L S I}} \subseteq \mathcal{L}(\mathrm{J} \cap \mathrm{ECom})$ follows immediately from the inclusion $\mathcal{L} \mathrm{SI} \subseteq \mathcal{L}(\mathrm{J} \cap \mathrm{ECom})$ and by Corollary 5.10 .

Consider the alphabet $A=\{a, b\}$ and the pseudowords $u=a^{\omega} b^{\omega} a^{\omega}$ and $v=a^{\omega} b^{\omega} a^{\omega} b^{\omega} a^{\omega}$ of $\bar{\Omega}_{A} \mathrm{~S}$. Since $u$ and $v$ have the same finite factors, the same set of finite prefixes, and the same set of finite suffixes, we have $\mathcal{L} S \mathrm{FI}$ $u=v$ (cf. [20]). As $u$ and $v$ are products of idempotents, it follows from Corollary $[5.6$ that $\overline{\mathcal{L S I}} \models u=v$.

Let $\alpha=a a, \beta=b b, \gamma=a b$ and $\delta=b a$. We have the factorizations

$$
\begin{aligned}
& \Phi_{1}(u)=\alpha^{\omega-1} \cdot \gamma \cdot \beta^{\omega-2} \cdot \delta \cdot \alpha^{\omega-1}, \\
& \Phi_{1}(v)=\alpha^{\omega-1} \cdot \gamma \cdot \beta^{\omega-2} \cdot \delta \cdot \alpha^{\omega-2} \cdot \gamma \cdot \beta^{\omega-2} \cdot \delta \cdot \alpha^{\omega-1} .
\end{aligned}
$$

Both factorizations are multiregularly organized with short ( $\cap \cap$ ECom)breaks. As $\mathrm{J} \cap \mathrm{ECom}$ is closed under bideterministic product, by Theorem 7.2 we have $\mathrm{J} \cap \mathrm{ECom} \not \models \Phi_{1}(u)=\Phi_{1}(v)$. This in turn implies that $(\mathrm{J} \cap \mathrm{ECom}) * \mathrm{D}_{1} \not \forall u=v$ by [1, Theorem 10.6.12]. Since $(\mathrm{J} \cap \mathrm{ECom}) * \mathrm{D}_{1}$ is contained in $\mathcal{L}(\mathrm{J} \cap \mathrm{ECom})$, we conclude that $\mathcal{L}(\mathrm{J} \cap \mathrm{ECom}) \not \models u=v$.

Recall that the Brandt semigroup $B_{2}$ belongs to $\mathrm{V}$ if and only if $\mathrm{V} \nsubseteq \mathrm{DS}$. 
Lemma 10.2. Let $\mathrm{V}$ be a semigroup pseudovariety containing $B_{2}$. If $\mathrm{V}$ is multiregularly based, then $g \vee$ is multiregularly based.

Proof. In [5, Theorem 5.9] it is stated that if $\mathrm{V}=\llbracket u_{i}=v_{i} \mid i \in \mathcal{I} \rrbracket$ for a family $\left(u_{i}=v_{i}\right)_{i \in \mathcal{I}}$ of pseudoidentities, then $g \mathrm{~V}=\llbracket\left(u_{i}=v_{i} ; A_{u_{i}}\right) \mid i \in \mathcal{I} \rrbracket$, where $A_{u_{i}}$ is a certain graph canonically built from $u_{i}$, with the pseudowords $u_{i}$ and $v_{i}$ seen as pseudopaths in $\bar{\Omega}_{A_{u_{i}}} \mathrm{Sd}$. Hence, if the pseudoword $w_{i}$ is a regular factor of $u_{i}$, say $w_{i}=w_{i} x_{i} w_{i}$ for some pseudoword $x_{i}$, we have in particular $g \bigvee \models\left(w_{i}=w_{i} x_{i} w_{i} ; A_{u_{i}}\right)$, and the result follows.

Recall that, for a positive integer $k$, the pseudovariety of semigroups $\mathrm{D}_{k}$ is defined by $y x_{1} \cdots x_{k}=x_{1} \cdots x_{k}$, and $\mathrm{D}=\bigcup_{k>1} \mathrm{D}_{k}$.

Proposition 10.3. If $g \mathrm{~V}$ is multiregularly based, then $\mathrm{V} * \mathrm{D}$ and $\mathrm{V} * \mathrm{D}_{k}$ are multiregularly based, for every $k \geq 1$.

Proof. Let $\Sigma$ be a basis of $g \vee$ such that, for each $(u=v ; A) \in \Sigma$, both $u$ and $v$ are multiregular edges of $\bar{\Omega}_{A} \mathrm{Sd}$. For each pseudoidentity $P$ in $\Sigma$, let $\mathcal{H}_{P}$ be the set of continuous semigroupoid homomorphisms from $\bar{\Omega}_{A} \mathrm{Sd}$ onto free profinite semigroups $\bar{\Omega}_{n} \mathrm{~S}$, with $n$ running the positive integers.

By [5, Theorem 3.2], we can pick a certain $\varepsilon_{P} \in \mathcal{H}_{P}$, for each pseudoidentity $P=(u=v)$ in $\Sigma$, in such a way that

$$
\mathrm{V} * \mathrm{D}=\bigcap_{P=(u=v) \in \Sigma} \llbracket \varepsilon_{P}(u)=\varepsilon_{P}(v) \rrbracket .
$$

Since $u$ and $v$ are multiregular edges, their homomorphic images $\varepsilon_{P}(u)$ and $\varepsilon_{P}(v)$ are multiregular pseudowords, establishing the proposition for $\mathbf{V} * \mathrm{D}$.

Concerning $\mathrm{V} * \mathrm{D}_{k}$, we make a proof by induction on $k \geq 1$. Beginning with case $k=1$, we apply to $\mathrm{V} * \mathrm{D}_{1}$ the Almeida-Weil basis theorem [12, Theorem 5.3], as follows. For each $\varepsilon \in \mathcal{H}_{P}$, let $\mathcal{F}_{P, \varepsilon}$ be the set of families $\pi=\left(\pi_{q}\right)_{q \in V(A)}$ of elements of $\left(\bar{\Omega}_{n} S\right)^{1}$ for which one has $\mathrm{D}_{1} \models \varepsilon(s)=\pi_{\omega s}$ (actually, in a literal application of the Almeida-Weil basis theorem one has first $\mathrm{D}_{1} \models \pi_{\alpha s} \varepsilon(s)=\pi_{\omega s}$, clearly equivalent to $\mathrm{D}_{1} \models \varepsilon(s)=\pi_{\omega s}$, as $\varepsilon(s)$ is a nonempty pseudoword). The Almeida-Weil basis theorem gives

$$
\mathrm{V} * \mathrm{D}_{1}=\bigcap_{P \in \Sigma} \bigcap_{\varepsilon \in \mathcal{H}_{P}} \bigcap_{\pi \in \mathcal{F}_{P, \varepsilon}} \llbracket \pi_{\alpha u} \varepsilon(u)=\pi_{\alpha u} \varepsilon(v) \mid P=(u=v) \rrbracket .
$$

Fixed $\pi \in \mathcal{F}_{P, \varepsilon}$, consider the family $\pi^{\prime}=\left(\mathrm{t}_{1}\left(\pi_{q}\right)\right)_{q \in V(A)}$. Note that we have $\mathrm{D}_{1} \models \varepsilon(s)=\mathrm{t}_{1}\left(\pi_{\omega s}\right)$, whence $\pi^{\prime} \in \mathcal{F}_{P, \varepsilon}$. On the other hand, the pseudoidentity $\pi_{\alpha u} \varepsilon(u)=\pi_{\alpha u} \varepsilon(v)$ is clearly a consequence of the pseudoidentity $\mathrm{t}_{1}\left(\pi_{\alpha u}\right) \varepsilon(u)=\mathrm{t}_{1}\left(\pi_{\alpha u}\right) \varepsilon(v)$. We conclude that we actually have

$$
\mathrm{V} * \mathrm{D}_{1}=\bigcap_{P \in \Sigma} \bigcap_{\varepsilon \in \mathcal{H}_{P}} \bigcap_{\pi \in \mathcal{F}_{P, \varepsilon}} \llbracket \mathrm{t}_{1}\left(\pi_{\alpha u}\right) \varepsilon(u)=\mathrm{t}_{1}\left(\pi_{\alpha u}\right) \varepsilon(v) \mid P=(u=v) \rrbracket .
$$

Therefore, it suffices to check that, fixed $P=(u=v) \in \Sigma$ and $\pi \in \mathcal{F}_{P, \varepsilon}$, both $\mathrm{t}_{1}\left(\pi_{\alpha u}\right) \varepsilon(u)$ and $\mathrm{t}_{1}\left(\pi_{\alpha u}\right) \varepsilon(v)$ are multiregular. By hypothesis, there is a factorization $u=w u^{\prime}$ such that $w$ is a regular edge of $\bar{\Omega}_{A} \mathrm{Sd}$ and $u^{\prime}$ is a 
product of regular edges of $\bar{\Omega}_{A} \mathrm{Sd}$. Let $z \in \bar{\Omega}_{A} \mathrm{Sd}$ be such that $w=w z w$. Take $s \in E(A)$ with $z=z^{\prime} s$, for some (possibly empty) pseudopath $z^{\prime}$. Since $\pi^{\prime} \in \mathcal{F}_{P, \varepsilon}$, and noting that $\omega s=\alpha w=\alpha u$, we have $\varepsilon(s) \leq_{\mathcal{L}} \mathrm{t}_{1}\left(\pi_{\alpha u}\right)$. It follows that $\varepsilon(w)=\varepsilon(w z w) \leq_{\mathcal{L}} \varepsilon(s w) \leq_{\mathcal{L}} \mathrm{t}_{1}\left(\pi_{\alpha u}\right) \varepsilon(w) \leq_{\mathcal{L}} \varepsilon(w)$, showing that $\mathrm{t}_{1}\left(\pi_{\alpha u}\right) \varepsilon(w)$ is $\mathcal{L}$-equivalent to the regular pseudoword $\varepsilon(w)$. Therefore, $\mathrm{t}_{1}\left(\pi_{\alpha u}\right) \varepsilon(u)=\mathrm{t}_{1}\left(\pi_{\alpha u}\right) \varepsilon(w) \varepsilon\left(u^{\prime}\right)$ is a product of regular pseudowords. Symmetrically, as $\alpha u=\alpha v$, the pseudoword $\mathrm{t}_{1}\left(\pi_{\alpha u}\right) \varepsilon(v)$ is also a product of regular pseudowords. This shows the base step of the induction.

We proceed with the inductive step. If $k>1$, then $\mathrm{D}_{k}=\mathrm{D}_{k-1} * \mathrm{D}_{1}$ holds (cf. [1, Lemma 4.1]), thus $\mathrm{V} * \mathrm{D}_{k}=\left(\mathrm{V} * \mathrm{D}_{k-1}\right) * \mathrm{D}_{1}$. By the induction hypothesis, $\mathrm{V} * \mathrm{D}_{k-1}$ is multiregularly based. Since $B_{2} \in \mathrm{SI} * \mathrm{D}_{1}$ (cf. [5, Section 4]), it then follows from Lemma 10.2 that $g\left(\mathrm{~V} * \mathrm{D}_{\mathrm{k}-1}\right)$ is multiregularly based. This reduces to the base case, thus proving that $\mathrm{V} * \mathrm{D}_{k}$ is multiregularly based.

Corollary 10.4. Suppose that $\mathrm{V}$ is a semigroup pseudovariety belonging to one of the intervals [SI, DS $\cap \mathrm{RS}]$ or [J, DS]. If $\mathrm{V}$ is closed under bideterministic product, then so is each of the pseudovarieties $\mathrm{V} * \mathrm{D}$ and $\mathrm{V} * \mathrm{D}_{k}$, for every $k \geq 1$. Therefore, if $\mathrm{V}$ belongs to one of the intervals [SI, DS $\cap \mathrm{RS}$ ] or [J, DS], the inclusions $\overline{\mathrm{V} * \mathrm{D}} \subseteq \overline{\mathrm{V}} * \mathrm{D}$ and $\overline{\mathrm{V} * \mathrm{D}_{k}} \subseteq \overline{\mathrm{V}} * \mathrm{D}_{k}$ hold.

Proof. If $\mathrm{V}$ is closed under bideterministic product, then $g \mathrm{~V}$ is multiregularly based by Corollary 8.11. Therefore, the pseudovarieties $\mathrm{V} * \mathrm{D}$ and $\mathrm{V} * \mathrm{D}_{k}$ are multiregularly based, by Proposition 10.3 , which implies by Proposition 5.8 that they are closed under bideterministic product.

Example 10.5. The pseudovariety DG $=\llbracket(x y)^{\omega}=(y x)^{\omega} \rrbracket$ is mutiregulary based, and therefore so is each of the pseudovarieties $D G * D$ and $D G * D_{k}$.

Remark 10.6. The existing proof that DG is local is a tour de force that does not depend on profinite methods [26]. Note that DG = IE $m$ SI, where $\mathrm{IE}=\llbracket x^{\omega}=y^{\omega} \rrbracket$, so that $\mathcal{L} \mathrm{DG}=\mathrm{IE}(m \mathcal{L} \mathrm{SI}$, as proved in [19, Appendix A]. Since $\mathrm{SI}$ is local, one has $\mathcal{L} \mathrm{SI}=\mathrm{SI} * \mathrm{D}=\mathrm{V}\left(B_{2}\right) * \mathrm{D}$, where $\mathrm{V}\left(B_{2}\right)$ is the semigroup pseudovariety generated by $B_{2}$. Moreover, thanks to [19, Theorem $4.2]$, the inclusion IE $m\left(\mathrm{~V}\left(B_{2}\right) * \mathrm{D}\right) \subseteq\left(\mathrm{IE} ; \mathrm{V}\left(B_{2}\right)\right) * \mathrm{D}$ holds. Therefore, we may hope for an alternative proof of the locality of DG consisting in showing that

$$
\mathrm{IE} / \mathrm{V} \mathrm{V}\left(B_{2}\right) \subseteq \mathrm{DG} * \mathrm{D}_{1}
$$

This inclusion is indeed true, but the arguments we have for its justification depend on the locality of DG. In this context, Example 10.5] is relevant, since it reduces the problem to the search of a "profinite" proof of the inclusion (10.1) to showing that IE $\mathrm{m} \mathrm{V}\left(B_{2}\right) \models \pi=\rho$ whenever $\pi$ and $\rho$ are multiregular pseudowords such that $\mathrm{DG} * \mathrm{D}_{1} \models \pi=\rho$.

For pseudovarieties not contained in DS, we have the following proposition. We omit the proof, because it is an exercise using the ideas in the 
proof of [18, Lemma 3.11] and none of the equational techniques that are the subject of this paper.

Proposition 10.7. Suppose that $\mathrm{V}$ is a semigroup pseudovariety such that $B_{2} \in \mathrm{V}$ and $\mathrm{D}_{1} \vee \mathrm{K}_{1} \subseteq \mathrm{V}$. If $\mathrm{V}$ is closed under bideterministic product, then so is $\mathrm{V} * \mathrm{D}_{k}$, for every $k \geq 1$.

We leave open the question of whether the condition $\mathrm{D}_{1} \vee \mathrm{K}_{1} \subseteq \mathrm{V}$ in Proposition 10.7 is really necessary. As one example that it might not be, take the pseudovariety ECom: on one hand, $B_{2} \in \mathrm{ECom}$ and $\mathrm{D}_{1} \nsubseteq \subseteq \mathrm{ECom}$, on the other hand $\mathrm{ECom} * \mathrm{D}_{k}$ is closed under bideterministic product for each $k$, by the combination of Lemma 10.2, Proposition 10.3 and Proposition 5.8 .

\section{REFERENCES}

[1] Almeida, J.: Finite Semigroups and Universal Algebra. World Scientific, Singapore (1995). English translation

[2] Almeida, J.: A syntactical proof of locality of DA. Int. J. Algebra Comput. 6, 165-177 (1996)

[3] Almeida, J.: Profinite semigroups and applications. In: V.B. Kudryavtsev, I.G. Rosenberg (eds.) Structural theory of automata, semigroups and universal algebra, pp. 1-45. Springer, New York (2005)

[4] Almeida, J., Azevedo, A.: On regular implicit operations. Portugal. Math. 50, 35-61 (1993)

[5] Almeida, J., Azevedo, A., Teixeira, L.: On finitely based pseudovarieties of the forms $\mathrm{V} * \mathrm{D}$ and $\mathrm{V} * \mathrm{D}_{n}$. J. Pure Appl. Algebra 146, 1-15 (2000)

[6] Almeida, J., Costa, A.: Infinite-vertex free profinite semigroupoids and symbolic dynamics. J. Pure Appl. Algebra 213, 605-631 (2009)

[7] Almeida, J., Costa, A.: Equidivisible pseudovarieties of semigroups. Publ. Math. Debrecen 90, 435-453 (2017)

[8] Almeida, J., Costa, A., Costa, J.C., Zeitoun, M.: The linear nature of pseudowords. Tech. rep. (2017). arXiv:1702.08083, submitted

[9] Almeida, J., Escada, A.: The globals of some subpseudovarieties of DA. Int. J. Algebra Comput. 14, 525-549 (2004)

[10] Almeida, J., Weil, P.: Reduced factorizations in free profinite groups and join decompositions of pseudovarieties. Int. J. Algebra Comput. 4, 375-403 (1994)

[11] Almeida, J., Weil, P.: Relatively free profinite monoids: an introduction and examples. In: J.B. Fountain (ed.) Semigroups, Formal Languages and Groups, vol. 466, pp. 73-117. Kluwer Academic Publ., Dordrecht (1995)

[12] Almeida, J., Weil, P.: Profinite categories and semidirect products. J. Pure Appl. Algebra 123, 1-50 (1998)

[13] Auinger, K.: Join decompositions of pseudovarieties involving semigroups with commuting idempotents. J. Pure Appl. Algebra 170(2-3), 115-129 (2002)

[14] Azevedo, A.: Operações implícitas sobre pseudovariedades de semigrupos. Aplicações. Ph.D. thesis, Universidade do Porto (1989)

[15] Branco, M.J.J.: On the Pin-Thérien expansion of idempotent monoids. Semigroup Forum 49, 329-334 (1994)

[16] Branco, M.J.J.: Two algebraic approaches to variants of the concatenation product. Theor. Comp. Sci. 369(1-3), 406-426 (2006)

[17] Branco, M.J.J.: Deterministic concatenation product of languages recognized by finite idempotent monoids. Semigroup Forum 74(3), 379-409 (2007)

[18] Costa, A.: Pseudovarieties defining classes of sofic subshifts closed under taking shift equivalent subshifts. J. Pure Appl. Algebra 209, 517-530 (2007) 
[19] Costa, A., Escada, A.: Some operators that preserve the locality of a pseudovariety of semigroups. Int. J. Algebra Comput. 23, 583-610 (2013)

[20] Costa, J.C.: Free profinite $\mathcal{R}$-trivial, locally idempotent and locally commutative semigroups. Semigroup Forum 58, 423-444 (1999)

[21] Eilenberg, S.: Automata, Languages and Machines, vol. B. Academic Press, New York (1976)

[22] Jiang, Z.: On the Pin-Thérien expansion of completely regular monoids. Semigroup Forum 60, 1-3 (2000)

[23] Jones, P.R.: Monoid varieties defined by $x^{n+1}=x$ are local. Semigroup Forum 47, 318-326 (1993)

[24] Jones, P.R.: Profinite categories, implicit operations and pseudovarieties of categories. J. Pure Appl. Algebra 109, 61-95 (1996)

[25] Jones, P.R., Szendrei, M.B.: Local varieties of completely regular monoids. J. Algebra 150, 1-27 (1992)

[26] Kadourek, J.: On the locality of the pseudovariety DG. J. Inst. Math. Jussieu 7, 93-180 (2008)

[27] Pin, J.E.: Propriétés syntactiques du produit non ambigu. In: Automata, languages and programming (Proc. Seventh Internat. Colloq., Noordwijkerhout, 1980), Lect. Notes in Comput. Sci., vol. 85, pp. 483-499. Springer, Berlin (1980)

[28] Pin, J.E.: Varieties of Formal Languages. Plenum, London (1986). English translation

[29] Pin, J.E.: Syntactic semigroups. In: G. Rozenberg, A. Salomaa (eds.) Handbook of Formal Languages, chap. 10. Springer (1997)

[30] Pin, J.E., Straubing, H., Thérien, D.: Locally trivial categories and unambiguous concatenation. J. Pure Appl. Algebra 52, 297-311 (1988)

[31] Pin, J.E., Thérien, D.: The bideterministic concatenation product. Int. J. Algebra Comput. 3, 535-555 (1993)

[32] Pin, J.E., Weil, P.: Profinite semigroups, Mal'cev products and identities. J. Algebra 182, 604-626 (1996)

[33] Reiterman, J.: The Birkhoff theorem for finite algebras. Algebra Universalis 14, 1-10 (1982)

[34] Rhodes, J., Steinberg, B.: The $q$-theory of finite semigroups. Springer Monographs in Mathematics. Springer (2009)

[35] Straubing, H.: Aperiodic homomorphisms and the concatenation product of recognizable sets. J. Pure Appl. Algebra 15, 319-327 (1979)

[36] Tilson, B.: Categories as algebra: an essential ingredient in the theory of monoids. J. Pure Appl. Algebra 48, 83-198 (1987)

[37] Trotter, P.G., Weil, P.: The lattice of pseudovarieties of idempotent semigroups and a non-regular analogue. Algebra Universalis 37, 491-526 (1997)

CMUC, Department of Mathematics, University of Coimbra, Apartado 3008, EC Santa Cruz, 3001-501 Coimbra, Portugal.

E-mail address: amgc@mat.uc.pt

CMUC, Department of Mathematics, University of Coimbra, Apartado 3008, EC Santa Cruz, 3001-501 Coimbra, Portugal.

E-mail address: apce@mat.uc.pt 